\title{
Discrete Subspace Multiwindow Gabor Frames and Their Duals
}

\author{
Yun-Zhang Li and Yan Zhang \\ College of Applied Sciences, Beijing University of Technology, Beijing 100124, China
}

Correspondence should be addressed to Yun-Zhang Li; yzlee@bjut.edu.cn

Received 2 March 2013; Accepted 7 May 2013

Academic Editor: Wenchang Sun

Copyright (c) 2013 Y.-Z. Li and Y. Zhang. This is an open access article distributed under the Creative Commons Attribution License, which permits unrestricted use, distribution, and reproduction in any medium, provided the original work is properly cited.

\begin{abstract}
This paper addresses discrete subspace multiwindow Gabor analysis. Such a scenario can model many practical signals and has potential applications in signal processing. In this paper, using a suitable Zak transform matrix we characterize discrete subspace mixed multi-window Gabor frames (Riesz bases and orthonormal bases) and their duals with Gabor structure. From this characterization, we can easily obtain frames by designing Zak transform matrices. In particular, for usual multi-window Gabor frames (i.e., all windows have the same time-frequency shifts), we characterize the uniqueness of Gabor dual of type I (type II) and also give a class of examples of Gabor frames and an explicit expression of their Gabor duals of type I (type II).
\end{abstract}

\section{Introduction}

Let $\mathscr{H}$ be a separable Hilbert space. An at most countable sequence $\left\{h_{i}\right\}_{i \in \mathscr{I}}$ in $\mathscr{H}$ is called a frame for $\mathscr{H}$ if there exist $0<A \leq B<\infty$ such that

$$
A\|f\|^{2} \leq \sum_{i \in \mathscr{I}}\left|\left\langle f, h_{i}\right\rangle\right|^{2} \leq B\|f\|^{2} \text { for } f \in \mathscr{H},
$$

where $A$ and $B$ are called frame bounds. The sequence $\left\{h_{i}\right\}_{i \in \mathscr{I}}$ is called a Bessel sequence in $\mathscr{H}$ if the right-hand side inequality in (1) holds. In this case $B$ is called a Bessel bound. A frame for $\mathscr{H}$ is said to be a Riesz basis if it ceases to be a frame for $\mathscr{H}$ whenever an arbitrary element is removed. And in this case, the frame bounds are also called Riesz bounds. The fundamentals of frames can be found in [1-4]. We denote by $\mathbb{Z}$ the set of integers, by $\mathbb{N}$ the set of positive integers, by $\left\{\mathbf{e}_{i}\right\}$ the vector in Euclidean spaces with the $i$ th component being 1 and others being zero, and by $\mathbb{N}_{\gamma}$ the set $\{0,1, \ldots, \gamma-1\}$ for $\gamma \in \mathbb{N}$. This paper addresses Gabor systems $G(\mathbf{g}, \mathbf{N}, \mathbf{M})$ of the form

$$
G(\mathbf{g}, \mathbf{N}, \mathbf{M})=\left\{E_{m / M} T_{n N_{l}} g_{l}: 1 \leq l \leq L, m \in \mathbb{N}_{M}, n \in \mathbb{Z}\right\},
$$

where $L$ is a fixed positive integer, $\mathbf{g}=\left(g_{1}, g_{2}, \ldots, g_{L}\right)$ with each $g_{l} \in l^{2}(\mathbb{Z}), \mathbf{N}=\left(N_{1}, N_{2}, \ldots, N_{L}\right)$ with each $N_{l} \in \mathbb{N}$, and $\mathbf{M}=\left(M_{1}, M_{2}, \ldots, M_{L}\right)$ with each $M_{l} \in \mathbb{N}$. Throughout this paper, we work under the following assumptions.

Assumption 1. L is a positive integer.
Assumption 2. $M_{1}=M_{2}=\cdots=M_{L}=M$, and $N_{l} / M=$ $p_{l} / q_{l}$ with $p_{l}$ and $q_{l}$ being relatively prime positive integers for $1 \leq l \leq L$.

We always denote by $N$ the least common multiple of $N_{l}$ with $1 \leq l \leq L$, by $\lambda_{l}$ the positive integer satisfying $N=$ $\lambda_{l} N_{l}$ for each $1 \leq l \leq L$, by $p$ and $q$ two relatively prime positive integers satisfying $N / M=p / q$, and by $Q$ the number $Q=q \sum_{l=1}^{L} \lambda_{l}$. Obviously, they are all uniquely determined by Assumptions 1 and 2.

Remark 3. We do not lose generality by making Assumptions 1 and 2. Let us check a general Gabor system of the form

$$
G(\mathbf{g}, \mathbf{N}, \mathbf{M})=\left\{E_{m_{l} / M_{l}} T_{n N_{l}} g_{l}: 1 \leq l \leq L, m_{l} \in \mathbb{N}_{M_{l}}, n \in \mathbb{Z}\right\}
$$

with $\mathbf{g}=\left(g_{1}, g_{2}, \ldots, g_{L}\right)$ satisfying $g_{l} \in l^{2}(\mathbb{Z}), \mathbf{N}=\left(N_{1}, N_{2}\right.$, $\left.\ldots, N_{L}\right)$, and $\mathbf{M}=\left(M_{1}, M_{2}, \ldots, M_{L}\right)\left(M_{1}, M_{2}, \ldots, M_{L}\right.$ are not necessarily all the same). Suppose $M$ is the greatest common divisor of $M_{1}, M_{2}, \ldots, M_{L}$, and $M_{l}=\alpha_{l} M$ with $\alpha_{l} \in \mathbb{N}$ for $1 \leq l \leq L$. Define $g_{l}^{\left(\beta_{l}\right)}(\cdot)=e^{2 \pi i\left(\beta_{l} / M_{l}\right) \cdot} g_{l}(\cdot)$ for $1 \leq l \leq L$ and $\beta_{l} \in \mathbb{N}_{\alpha_{l}}$. Then, by a simple computation, $G(\mathbf{g}, \mathbf{N}, \mathbf{M})$ is a frame for a closed subspace $\mathscr{M}$ of $l^{2}(\mathbb{Z})$ if and only if the Gabor system

$$
\left\{E_{m / M} T_{n N_{l}} g_{l}^{\left(\beta_{l}\right)}: 1 \leq l \leq L, \beta_{l} \in \mathbb{N}_{\alpha_{l}}, m \in \mathbb{N}_{M}, n \in \mathbb{Z}\right\}
$$


is a frame for $\mathscr{M}$. Therefore, the study of (3) is reduced to the study of (4).

We denote by $\mathscr{M}(\mathbf{g}, \mathbf{N}, \mathbf{M})$ the closed linear span of $G(\mathbf{g}, \mathbf{N}, \mathbf{M})$. A Gabor system $G(\mathbf{g}, \mathbf{N}, \mathbf{M})$ of the form (2) is called a mixed multi-window Gabor system if $L>1$ since $N_{1}, N_{2}, \ldots$ and $N_{L}$ are not necessarily all the same. (In particular, this system is the usual multi-window Gabor system when $N_{1}=N_{2}=\cdots=N_{L}$ ). It is called a subspace mixed multi-window Gabor frame if it is a frame for $\mathscr{M}(\mathbf{g}, \mathbf{N}, \mathbf{M})$ and $L>1$. Similarly, a Gabor system $G(\mathbf{g}, \mathbf{N}, \mathbf{M})$ is called a single-window Gabor system if $L=1$ and called a single-window subspace Gabor frame if it is a frame for $\mathscr{M}(\mathbf{g}, \mathbf{N}, \mathbf{M})$ and $L=1$.

For a Bessel sequence $G(\mathbf{g}, \mathbf{N}, \mathbf{M})$ in $l^{2}(\mathbb{Z})$ of the form (2), we define the associated synthesis operator $\mathscr{T}_{\mathrm{g}}: l^{2}\left(\mathbb{N}_{M} \times\right.$ $\left.\mathbb{Z}, \mathbb{C}^{L}\right) \rightarrow l^{2}(\mathbb{Z})$ by

$$
\mathscr{T}_{\mathbf{g}} c=\sum_{l=1}^{L} \sum_{m \in \mathbb{N}_{M}} \sum_{n \in \mathbb{Z}} c_{l, m, n} E_{m / M} T_{n N_{l}} g_{l}
$$

for $c=\left(c_{1}, c_{2}, \ldots, c_{L}\right) \in l^{2}\left(\mathbb{N}_{M} \times \mathbb{Z}, \mathbb{C}^{L}\right)$. Then it is a bounded operator, and its adjoint operator $\mathscr{T}_{\mathrm{g}}^{*}$ (so-called analysis operator) is given by

$$
\mathscr{T}_{\mathbf{g}}^{*} f=\left(c_{1}(f), c_{2}(f), \ldots, c_{L}(f)\right) \quad \text { for } f \in l^{2}(\mathbb{Z}),
$$

where $c_{l}(f)=\left\{\left\langle f, E_{m / M} T_{n N_{l}} g_{l}\right\rangle\right\}_{m \in \mathbb{N}_{M}, n \in \mathbb{Z}}$ for each $1 \leq l \leq$ $L$. For a Bessel sequence $G(\mathbf{h}, \mathbf{N}, \mathbf{M})$ in $l^{2}(\mathbb{Z})$ with $\mathbf{h}=\left(h_{1}, h_{2}\right.$, $\ldots, h_{L}$ ), we associate it with $\mathscr{T}_{\mathbf{h}}$ similarly. Write $\mathcal{S}_{\mathbf{h}, \mathbf{g}}=\mathscr{T}_{\mathbf{g}} \mathscr{T}_{\mathbf{h}}^{*}$; that is,

$$
\mathcal{S}_{\mathbf{h}, \mathbf{g}} f=\sum_{l=1}^{L} \sum_{m \in \mathbb{N}_{M}} \sum_{n \in \mathbb{Z}}\left\langle f, E_{m / M} T_{n N_{l}} h_{l}\right\rangle E_{m / M} T_{n N_{l}} g_{l}
$$

for $f \in l^{2}(\mathbb{Z})$. Let $G(\mathbf{g}, \mathbf{N}, \mathbf{M})$ be a frame for $\mathscr{M}(\mathbf{g}, \mathbf{N}, \mathbf{M})$ and let $G(\mathbf{h}, \mathbf{N}, \mathbf{M})$ be a Bessel sequence in $l^{2}(\mathbb{Z}) . G(\mathbf{h}, \mathbf{N}, \mathbf{M})$ is called an oblique Gabor dual for $G(\mathbf{g}, \mathbf{N}, \mathbf{M})$ if

$$
\mathcal{S}_{\mathbf{h}, \mathbf{g}} f=f \quad \text { for } f \in \mathscr{M}(\mathbf{g}, N, M) .
$$

Here it is not required that each $h_{l}$ belongs to $\mathscr{M}(\mathbf{g}, \mathbf{N}, \mathbf{M})$. In particular, an oblique Gabor dual $G(\mathbf{h}, \mathbf{N}, \mathbf{M})$ for $G(\mathbf{g}, \mathbf{N}, \mathbf{M})$ is said to be a Gabor dual of type $I$ for $G(\mathbf{g}, \mathbf{N}, \mathbf{M})$ if $h_{l} \in$ $\mathscr{M}(\mathbf{g}, \mathbf{N}, \mathbf{M})$ for each $1 \leq l \leq L$ and is said to be a Gabor dual of type II for $G(\mathbf{g}, \mathbf{N}, \mathbf{M})$ if $\operatorname{range}\left(\mathscr{T}_{\mathbf{h}}^{*}\right) \subset \operatorname{range}\left(\mathscr{T}_{\mathbf{g}}^{*}\right)$. These notions of duals are a generalization of the ones in $[5,6]$. They are borrowed from $[7,8]$ which dealt with Gabor frames in $L^{2}(\mathbb{R})$. For a Gabor dual of type II, it is not required to be in $\mathscr{M}(\mathrm{g}, \mathbf{N}, \mathbf{M})$, but a containment relation between the ranges of analysis operators is required. Observe that the canonical $\operatorname{daul} G\left(\mathcal{S}_{\mathbf{g}, \mathbf{g}}^{-1} \mathbf{g}, \mathbf{N}, \mathbf{M}\right)$ for $G(\mathbf{g}, \mathbf{N}, \mathbf{M})$ belongs to any one of the three Gabor duals.

In the past more than twenty years, the theory of frames has been growing rapidly. Gabor frames are a class of important frames among all kinds of frames. For continuous Gabor frames, single-window Gabor frames for $L^{2}(\mathbb{R})$ have been studied extensively [2, 3, 9, 10]; multi-window Gabor frames for $L^{2}(\mathbb{R})$ were firstly studied by Zibulski and Zeevi in [11], and then by others in [12-17]; single-window subspace (of $L^{2}(\mathbb{R})$ ) Gabor frames were studied in $[7,8,18-23]$. In digital signal processing, one usually encounters discrete signals instead of continuous ones. One can obtain discrete Gabor frames for $l^{2}(\mathbb{Z})$ via Gabor frames for $L^{2}(\mathbb{R})$ through sampling under certain additional assumptions [24-26], though these assumptions are artificial and too technical. So it should be more reasonable to consider Gabor frames in $l^{2}(\mathbb{Z})$ without referring to frames in $L^{2}(\mathbb{R})$. Intuitively, the general theory of discrete Gabor analysis is similar to the continuous case. Sometimes major differences occur. In 1989, Heil in [27] showed that while Gabor frames in the continuous case are bases only if they are generated by functions that are not smooth or have poor decay, it is possible in the discrete case to construct Gabor frames that are bases and are generated by sequences with good decay. The sampled Gaussian gives an example of such a signal. Also due to its good potential for digital signal processing, since then, Gabor analysis on $l^{2}(\mathbb{Z})$ has attracted many researchers (see $[2,3,24-34]$ and the references therein). In $[6,35]$, single-window Gabor frames on discrete periodic subsets of $\mathbb{Z}$ were investigated. It is well known that a single-window Gabor expansion is not enough to analyze the dynamic time-frequency contents of signals that contain a wide range of spatial and frequency components. A multi-window Gabor expansion is capable of extracting local frequencies in an adaptive manner, in which wide windows are responsible for slow-changing components and narrow windows are designed to extract transient and rapid-changing components of a signal. See [15] and the references therein for details. In addition, Example 38 and the arguments before it in the last section show that, for Gabor duals, multi-window Gabor frames behave differently from single-window ones. Motivated by the above works, we in this paper study subspace Gabor frame of the form (2) under Assumptions 1 and 2.

The rest of this paper is organized as follows. Section 2 is an auxiliary one to following sections. In this section, we introduce the notion of Zak transform matrix associated with a Gabor system of the form (2) and investigate its properties. In terms of Zak transform matrices, we in Section 3 characterize subspace multi-window Gabor frames (Riesz bases, orthonormal bases) and in Section 4 Gabor duals of type I (type II) for subspace multi-window Gabor frames. Section 5 focuses on subspace multi-window Gabor frames with all the windows having the same time-frequency shifts. We characterize the uniqueness of Gabor duals of type I (type II) and obtain a class of examples of subspace multiwindow Gabor frames (Riesz bases, orthonormal bases) and their Gabor duals of type I (type II) (see Theorems 36 and 37). 


\section{Zak Transforms and Zak Transform Matrices}

This section focuses on some properties of Zak transforms and Zak transform matrices, which is an auxiliary one to following sections. We start with some notations and notions. For $s, t \in \mathbb{N}$, we denote by $\mathscr{M}_{s, t}$ the set of $s \times t$ complex matrices. Given $\gamma \in \mathbb{N}$, two subsets $S_{1}$ and $S_{2}$ of $\mathbb{Z}$ are said to be $\gamma \mathbb{Z}$ congruent if there exists a sequence of subsets $\left\{S_{1, k}\right\}_{k \in \mathbb{Z}}$ of $S_{1}$ such that $\bigcup_{k \in \mathbb{Z}} S_{1, k}=S_{1}, S_{1, k_{1}} \cap S_{1, k_{2}}=\emptyset$ for $k_{1} \neq k_{2}$ in $\mathbb{Z}$ and that $S_{2}=\bigcup_{k \in \mathbb{Z}}\left(S_{1, k}+\gamma k\right),\left(S_{1, k_{1}}+\gamma k_{1}\right) \cap\left(S_{1, k_{2}}+\gamma k_{2}\right)=\emptyset$ for $k_{1} \neq k_{2}$ in $\mathbb{Z}$. If $S_{1}$ or $S_{2}$ is a finite set in addition, only finitely many $S_{1, k}$ in the above are nonempty, while the others are empty. For two vectors $x$ and $y$ in a Hilbert space $\mathscr{H}$, we always write their inner product as $\langle x, y\rangle$ instead of $\langle x, y\rangle_{\mathscr{H}}$ when it causes no confusions. Let $E$ be a finite set in $\mathbb{Z}$ and let $\Gamma \in \mathbb{N}$. We denote by $L^{2}\left(E \times[0,1), \mathbb{C}^{\Gamma}\right)$ the Hilbert space of vector-valued functions $f$ satisfying $f(j, \cdot) \in L^{2}\left([0,1), \mathbb{C}^{\Gamma}\right)$ for each $j \in E$ endowed with the inner product

$$
\langle f, \tilde{f}\rangle=\sum_{j \in E} \int_{0}^{1}\langle f(j, v), \tilde{f}(j, v)\rangle d v
$$

for $f, \tilde{f} \in L^{2}\left(E \times[0,1), \mathbb{C}^{\Gamma}\right)$, where $\langle f(j, v), \tilde{f}(j, v)\rangle$ denotes their inner product in $\mathbb{C}^{\Gamma}$; by $l^{2}\left(E \times \mathbb{Z}, \mathbb{C}^{\Gamma}\right)$ the Hilbert space of sequences $c=\left(c_{1}, c_{2}, \ldots, c_{\Gamma}\right)$ satisfying $c_{\gamma} \in l^{2}(E \times \mathbb{Z})$ endowed with the inner product

$$
\langle c, \widetilde{c}\rangle=\sum_{\gamma=1}^{\Gamma}\left\langle c_{\gamma}, \widetilde{c}_{\gamma}\right\rangle
$$

for $c, \widetilde{c} \in l^{2}\left(E \times \mathbb{Z}, \mathbb{C}^{\Gamma}\right)$, where $\left\langle c_{\gamma}, \widetilde{c}_{\gamma}\right\rangle$ denotes their inner product in $l^{2}(E \times \mathbb{Z})$ and by $l_{0}\left(E \times \mathbb{Z}, \mathbb{C}^{\Gamma}\right)$ the set of sequences $c=\left(c_{1}, c_{2}, \ldots, c_{\Gamma}\right)$ with each $c_{\gamma}$ being a finitely supported sequence defined on $E \times \mathbb{Z}$. For simplicity, we write $L^{2}(E \times$ $[0,1))$ for $L^{2}\left(E \times[0,1), \mathbb{C}^{\Gamma}\right), l^{2}(E \times \mathbb{Z})$ for $l^{2}\left(E \times \mathbb{Z}, \mathbb{C}^{\Gamma}\right)$, and $l_{0}(E \times \mathbb{Z})$ for $l_{0}\left(E \times \mathbb{Z}, \mathbb{C}^{\Gamma}\right)$ when $\Gamma=1$. $f$ by

For $f \in l^{2}(\mathbb{Z})$, define the discrete Zak transform $\mathscr{Z}_{q N} f$ of

$$
\left(\mathscr{Z}_{q N} f\right)(j, v):=\sum_{\ell \in \mathbb{Z}} f(j+\ell q N) e^{2 \pi i \ell v}
$$

for $j \in \mathbb{Z}$ and a.e. $v \in \mathbb{R}$. It is easy to check that $\mathscr{Z}_{q N}$ has quasi-periodicity:

$$
\left(\mathscr{Z}_{q N} f\right)(j+k q N, v+\ell)=e^{-2 \pi i k v}\left(\mathscr{Z}_{q N} f\right)(j, v)
$$

for $k, \ell, j \in \mathbb{Z}$ and a.e. $v \in \mathbb{R}$.

Definition 4. For $\mathbf{g}=\left(g_{1}, g_{2}, \ldots, g_{L}\right)$ with each $g_{l} \in l^{2}(\mathbb{Z})$, we associate it with a matrix-valued function $\Psi_{\mathbf{g}}(j, v): \mathbb{Z} \times \mathbb{R} \rightarrow$ $\mathscr{M}_{\mathrm{Q}, p}$ by

$$
\Psi_{\mathbf{g}}(j, v)=\left(\begin{array}{c}
G_{1}(j, v) \\
G_{2}(j, v) \\
\vdots \\
G_{L}(j, v)
\end{array}\right) \text {, }
$$

where $Q:=q \sum_{l=1}^{L} \lambda_{l}$ and $G_{l}(j, v)$ is a block matrix of the form

$$
G_{l}(j, v)=\left(\begin{array}{c}
\mathscr{G}_{l}(j, v) \\
\mathscr{G}_{l}\left(j-N_{l}, v\right) \\
\vdots \\
\mathscr{G}_{l}\left(j-\left(\lambda_{l}-1\right) N_{l}, v\right)
\end{array}\right)
$$

with $\mathscr{G}_{l}(j, v): \mathbb{Z} \times \mathbb{R} \rightarrow \mathscr{M}_{q, p}$ for $1 \leq l \leq L$ and

$$
\mathscr{G}_{l}(j, v)_{r, k}=\mathscr{Z}_{q N} g_{l}(j-r N+k M, v)
$$

for $r \in \mathbb{N}_{q}, k \in \mathbb{N}_{p}$

By the quasi-periodicity of $\mathscr{Z}_{q N}$, an arbitrary $f \in l^{2}(\mathbb{Z})$ is uniquely determined by the values of $\left(\mathscr{Z}_{q N} f\right)(\cdot, \cdot)$ on $S \times$ $[0,1)$ with $S$ being a set $q N \mathbb{Z}$ congruent to $\mathbb{N}_{q N}$. So an arbitrary function $F \in L^{2}(S \times[0,1))$ determines a unique $f \in l^{2}(\mathbb{Z})$ by

$$
\left(\mathscr{Z}_{\mathrm{qN}} f\right)(j, v)=F(j, v) \quad \text { for }(j, v) \in S \times[0,1) .
$$

Let us make another assumption that $N_{1}=N_{2}=\cdots=N_{L}$ in Definition 4. Then $\Psi_{\mathbf{g}}(j, v)$ is an $L q \times p$-matrix-valued function of the form

$$
\begin{gathered}
\Psi_{\mathbf{g}}(j, v)=\left(\begin{array}{c}
\mathscr{G}_{1}(j, v) \\
\mathscr{G}_{2}(j, v) \\
\vdots \\
\mathscr{G}_{L}(j, v)
\end{array}\right), \\
\mathscr{G}_{l}(j, v)_{r, k}=\mathscr{Z}_{q N} g_{l}(j-r N+k M, v)
\end{gathered}
$$

for $1 \leq l \leq L, r \in \mathbb{N}_{q}$, and $k \in \mathbb{N}_{p}$. Also observe that $\mathbb{N}_{M / q}-N \mathbb{N}_{q}+M \mathbb{N}_{p}$ is $q N \mathbb{Z}$ congruent to $\mathbb{N}_{q N}$. We have if $N_{1}=N_{2}=\cdots=N_{L}$, then an arbitrary function $M(j, v)$ : $\mathbb{N}_{M / q} \times[0,1) \rightarrow \mathscr{M}_{L q, p}$ with all entries in $L^{2}\left(\mathbb{N}_{M / q} \times[0,1)\right)$ determines a unique $\mathbf{g}=\left(g_{1}, g_{2}, \ldots, g_{L}\right)$ with each $g_{l} \in l^{2}(\mathbb{Z})$ by

$$
\Psi_{\mathbf{g}}(j, v)=M(j, v) \quad \text { for } j \in \mathbb{N}_{M / q} \text {, a.e. } v \in[0,1) \text {. }
$$

But if $N_{l}, 1 \leq l \leq L$, are not all the same, a function $M(j, v)$ : $\mathbb{N}_{M / q} \times[0,1) \rightarrow \mathscr{M}_{Q, p}$ with all entries in $L^{2}\left(\mathbb{N}_{M / q} \times[0,1)\right)$ does not necessarily determine a $\mathbf{g}$. We show it by an example. 
In this case, there exists $1 \leq l \leq L$ such that $\lambda_{l}>1$. We may as well assume that $\lambda_{1}>1$. Choose

$$
\begin{gathered}
M(j, v)=\left(\begin{array}{c}
M_{1}(j, v) \\
M_{2}(j, v) \\
\vdots \\
M_{L}(j, v)
\end{array}\right), \\
M_{l}(j, v)=\left(\begin{array}{c}
M_{l, 0}(j, v) \\
M_{l, 1}(j, v) \\
\vdots \\
M_{l, \lambda_{l}-1}(j, v)
\end{array}\right)
\end{gathered}
$$

for $1 \leq l \leq L$ and $(j, v) \in \mathbb{N}_{M / q} \times[0,1)$ such that every entry of $M(j, v)$ belongs to $L^{2}\left(\mathbb{N}_{M / q} \times[0,1)\right)$ and that

$$
\begin{array}{r}
M_{1,0}(j, v) \neq 0, \quad M_{1,1}(j, v)=0 \\
\text { for }(j, v) \in \mathbb{N}_{M / q} \times[0,1) .
\end{array}
$$

Suppose there exists $\mathbf{g}$ such that $\Psi_{\mathbf{g}}(j, v)=M(j, v)$ for $j \in$ $\mathbb{N}_{M / q}$ and a.e. $v \in[0,1)$. Then

$$
\begin{gathered}
\mathscr{G}_{1}(j, v)=M_{1,0}(j, v), \\
\mathscr{G}_{1}\left(j-N_{1}, v\right)=M_{1,1}(j, v)
\end{gathered}
$$

for $j \in \mathbb{N}_{M / q}$ and a.e. $v \in[0,1)$, where

$$
\begin{gathered}
\mathscr{G}_{1}(j, v)_{r, k}=\mathscr{Z}_{q N} g_{1}(j-r N+k M, v), \\
\mathscr{G}_{1}\left(j-N_{1}, v\right)_{r, k}=\mathscr{Z}_{q N} g_{1}\left(j-N_{1}-r N+k M, v\right)
\end{gathered}
$$

for $(r, k) \in \mathbb{N}_{q} \times \mathbb{N}_{p}$. Since the sets $\mathbb{N}_{M / q}-N \mathbb{N}_{q}+M \mathbb{N}_{p}$ and $\mathbb{N}_{M / q}-N \mathbb{N}_{q}+M \mathbb{N}_{p}-N_{1}$ are both $q N \mathbb{Z}$ congruent to $\mathbb{N}_{q N}$, we have $\mathscr{Z}_{q N} g_{1}\left(j_{0}, v\right) \neq 0$ for some $j_{0} \in \mathbb{Z}$ and $v \in E \subset[0,1)$ with $|E|>0$ by (20), (21), and the quasi-periodicity of $\mathscr{Z}_{q N}$, while $\mathscr{Z}_{q N} g_{1}(j, v)=0$ for $j \in \mathbb{Z}$ and a.e. $v \in[0,1)$ by $(20),(22)$, and the quasi-periodicity of $\mathscr{Z}_{q N}$. This is a contradiction. Therefore, we must be careful when we define $\mathbf{g}$ by a function $M(j, v): \mathbb{N}_{M / q} \times[0,1) \rightarrow \mathscr{M}_{\mathrm{Q}, p}$ if $N_{l}, 1 \leq l \leq L$, are not all the same.

Definition 5. Define $\mathbf{Z}_{q N}: l^{2}(\mathbb{Z}) \rightarrow L^{2}\left(\mathbb{N}_{M} \times[0,1), \mathbb{C}^{p}\right)$ by

$$
\mathbf{Z}_{q N} f(j, v)=\left(\begin{array}{c}
\mathscr{Z}_{q N} f(j, v) \\
\mathscr{Z}_{q N} f(j+M, v) \\
\vdots \\
\mathscr{Z}_{q N} f(j+(p-1) M, v)
\end{array}\right)
$$

By the quasi-periodicity of $\mathscr{E}_{q N}$ and [35, Theorem 2.1], we have the following lemmas.

Lemma 6. $\mathrm{Z}_{q N}$ is a unitary operator from $l^{2}(\mathbb{Z})$ onto $L^{2}\left(\mathbb{N}_{M} \times\right.$ $\left.[0,1), \mathbb{C}^{p}\right)$, and $\mathscr{Z}_{q N}$ is a unitary operator from $l^{2}(\mathbb{Z})$ onto $L^{2}(E \times[0,1))$ for an arbitrary subset $E$ of $\mathbb{Z}$ which is $q N \mathbb{Z}$ congruent to $\mathbb{N}_{q N}$.

Definition 7. Define the Fourier transform $\mathscr{F}: l^{2}\left(\mathbb{N}_{M} \times \mathbb{Z}\right) \rightarrow$ $L^{2}\left(\mathbb{N}_{M} \times[0,1)\right)$ by

$$
\mathscr{F} c(j, v)=\frac{1}{\sqrt{M}} \sum_{m \in \mathbb{N}_{M}} \sum_{n \in \mathbb{Z}} c_{m, n} e^{2 \pi i(m / M) j} e^{2 \pi i n v}
$$

for $c \in l^{2}\left(\mathbb{N}_{M} \times \mathbb{Z}\right), j \in \mathbb{N}_{M}$, and a.e. $v \in[0,1)$.

Definition 8. Define $\mathscr{J}: l^{2}\left(\mathbb{N}_{M} \times \mathbb{Z}, \mathbb{C}^{L}\right) \rightarrow L^{2}\left(\mathbb{N}_{M} \times[0,1)\right.$, $\left.\mathbb{C}^{Q}\right)$ by

$$
\mathscr{J}_{c}(j, v)=\mathscr{C}(j, v)
$$

for $c=\left(c_{1}, c_{2}, \ldots, c_{L}\right) \in l^{2}\left(\mathbb{N}_{M} \times \mathbb{Z}, \mathbb{C}^{L}\right), j \in \mathbb{N}_{M}$, and a.e. $v \in \mathbb{R}$, where

$$
\begin{aligned}
& \mathscr{C}(j, v)=\left(\begin{array}{c}
\mathscr{C}_{1}(j, v) \\
\mathscr{C}_{2}(j, v) \\
\vdots \\
\mathscr{C}_{L}(j, v)
\end{array}\right) \\
& \mathscr{C}_{l}(j, v)=\left(\begin{array}{c}
\mathscr{C}_{l}^{(0)}(j, v) \\
\mathscr{C}_{l}^{(1)}(j, v) \\
\vdots \\
\mathscr{C}_{l}^{\left(\lambda_{l}-1\right)}(j, v)
\end{array}\right) \text {, } \\
& \mathscr{C}_{l}^{\left(\tau_{l}\right)}(j, v)=\left(\begin{array}{c}
\sum_{m \in \mathbb{N}_{M}} \sum_{n \in \mathbb{Z}} c_{l, m, n q \lambda_{l}+\tau_{l}} e^{2 \pi i(m / M) j} e^{2 \pi i n v} \\
\sum_{m \in \mathbb{N}_{M}} \sum_{n \in \mathbb{Z}} c_{l, m,(n q+1) \lambda_{l}+\tau_{l}} e^{2 \pi i(m / M) j} e^{2 \pi i n v} \\
\vdots \\
\sum_{m \in \mathbb{N}_{M}} \sum_{n \in \mathbb{Z}} c_{l, m,(n q+q-1) \lambda_{l}+\tau_{l}} e^{2 \pi i(m / M) j} e^{2 \pi i n v}
\end{array}\right)
\end{aligned}
$$

for $1 \leq l \leq L$ and $\tau_{l} \in \mathbb{N}_{\lambda_{l}}$.

Similarly, for an arbitrary $d \in l^{2}\left(\mathbb{N}_{M} \times \mathbb{Z}, \mathbb{C}^{L}\right)$, we associate it with $\mathscr{D}(j, v)$.

Lemma 9. (i) $\mathscr{F}$ is a unitary operator from $l^{2}\left(\mathbb{N}_{M} \times \mathbb{Z}\right)$ onto $L^{2}\left(\mathbb{N}_{M} \times[0,1)\right)$;

(ii) $(1 / \sqrt{M}) \mathscr{J}$ is a unitary operator from $l^{2}\left(\mathbb{N}_{M} \times \mathbb{Z}, \mathbb{C}^{L}\right)$ onto $L^{2}\left(\mathbb{N}_{M} \times[0,1), \mathbb{C}^{\mathrm{Q}}\right)$. 
Proof. Since $\left\{(1 / \sqrt{M}) e^{2 \pi i(m / M) j} e^{2 \pi i n v}: m \in \mathbb{N}_{M}, n \in \mathbb{Z}\right\}$ is an orthonormal basis for $L^{2}\left(\mathbb{N}_{M} \times[0,1)\right)$, we have (i). (ii) is an immediate consequence of (i).

Lemma 10. For $\mathbf{g}=\left(g_{1}, g_{2}, \ldots, g_{L}\right)$ with $g_{l} \in l^{2}(\mathbb{Z})$ for $1 \leq$ $l \leq L$, one has

(i) $\mathscr{Z}_{q N}\left(E_{m / M} T_{n q N+\tau_{l} N_{l}+r N} g_{l}\right)(j, v)=e^{2 \pi i(m / M) j} e^{2 \pi i n v}$ $\mathscr{Z}_{q N} g_{l}\left(j-\tau_{l} N_{l}-r N, v\right)$ for $r \in \mathbb{N}_{q}$;

(ii)

$$
\mathbf{Z}_{q N} f(j, v)=\overline{\Psi_{\mathbf{g}}^{*}(j, v)} \mathscr{J}_{c}(j, v)
$$

for $f=\mathscr{T}_{\mathrm{g}} c$ with $c \in l_{0}\left(\mathbb{N}_{M} \times \mathbb{Z}, \mathbb{C}^{L}\right)$, where $\mathscr{T}_{\mathrm{g}} c$ is as in (5).

Proof. (i) can be obtained by a direct computation. Applying (i), we have

$$
\begin{gathered}
\mathscr{Z}_{q N} f(j, v) \\
=\mathscr{Z}_{q N}\left(\sum_{l=1}^{L} \sum_{\tau_{l} \in \mathbb{N}_{\lambda_{l}}} \sum_{r \in \mathbb{N}_{q}} \sum_{m \in \mathbb{N}_{M}} \sum_{n \in \mathbb{Z}} c_{l, m,(n q+r) \lambda_{l}+\tau_{l}}\right. \\
\left.\times E_{m / M} T_{n q N+\tau_{l} N_{l}+r N} g_{l}\right) \\
=\sum_{l=1}^{L} \sum_{\tau_{l} \in \mathbb{N}_{\lambda_{l}}} \sum_{r \in \mathbb{N}_{q}} \mathscr{C}_{l}^{\left(\tau_{l}\right)}(j, v)_{r} \mathscr{Z}_{q N} g_{l}\left(j-\tau_{l} N_{l}-r N, v\right)
\end{gathered}
$$

for $(j, v) \in \mathbb{Z} \times \mathbb{R}$. This implies (ii).

Remark 11. When $G(\mathbf{g}, \mathbf{N}, \mathbf{M})$ in Lemma 10 is a Bessel sequence in $l^{2}(\mathbb{Z})$, by the same procedure as the above we can prove (28) holds for $f=\mathscr{T}_{\mathrm{g}} c$ with $c \in l^{2}\left(\mathbb{N}_{M} \times \mathbb{Z}, \mathbb{C}^{L}\right)$.

Lemma 12. For $\mathbf{g}=\left(g_{1}, g_{2}, \ldots, g_{L}\right)$ with $g_{l} \in l^{2}(\mathbb{Z})$ for $1 \leq$ $l \leq L$, one has

$$
\Psi_{\mathbf{g}}\left(j+\frac{M}{q} n, v\right)=e^{-2 \pi i m_{n} v} \mathscr{C}_{n}(v) \Psi_{\mathbf{g}}(j, v) \mathscr{D}_{n}(v)
$$

for $j \in \mathbb{Z}, n=k_{n} q+\left(m_{n} q-r_{n}\right) p$ with $\left(k_{n}, r_{n}, m_{n}\right) \in \mathbb{N}_{p} \times \mathbb{N}_{q} \times$ $\mathbb{Z}$, and a.e. $v \in \mathbb{R}$, where $\mathscr{D}_{n}(v)=\left(\begin{array}{cc}0 & e^{-2 \pi i v} I_{k_{n}} \\ I_{p-k_{n}} & 0\end{array}\right), \mathscr{C}_{n}(v)=$ $\operatorname{diag}\left(\mathscr{C}_{1, n}(v), \mathscr{C}_{2, n}(v), \ldots, \mathscr{C}_{L, n}(v)\right)$, and $\mathscr{C}_{l, n}(v)$ denotes the block matrix (with $\lambda_{l}$ blocks) of the form $\operatorname{diag}\left(C_{n}(v)\right.$, $\left.C_{n}(v), \ldots, C_{n}(v)\right)$ with $C_{n}(v)=\left(\begin{array}{cc}0 & I_{q-r_{n}} \\ e^{2 \pi i v} I_{r_{n}} & 0\end{array}\right)$.

Proof. By [6, Lemma 5], we have

$$
\mathscr{G}_{l}\left(j+\frac{M}{q} n, v\right)=e^{-2 \pi i m_{n} v} C_{n}(v) \mathscr{G}_{l}(j, v) \mathscr{D}_{n}(v)
$$

for $1 \leq l \leq L, j \in \mathbb{Z}, n=k_{n} q+\left(m_{n} q-r_{n}\right) p$ with $\left(k_{n}, r_{n}, m_{n}\right) \in$ $\mathbb{N}_{p} \times \mathbb{N}_{q} \times \mathbb{Z}$, and a.e. $v \in \mathbb{R}$. This leads to the lemma.

\section{Frame Characterization}

This section is devoted to characterization of subspace Gabor frames $G(\mathbf{g}, \mathbf{N}, \mathbf{M})$ of the form (2).

Theorem 13. For any $\mathbf{g}=\left(g_{1}, g_{2}, \ldots, g_{L}\right)$ with each $g_{l} \in$ $l^{2}(\mathbb{Z})$, the following are equivalent.

(i) $G(\mathbf{g}, \mathbf{N}, \mathbf{M})$ is a Bessel sequence in $l^{2}(\mathbb{Z})$ with Bessel bound $B$.

(ii) $\Psi_{\mathbf{g}}(j, v) \Psi_{\mathbf{g}}^{*}(j, v) \leq(B / M) I$ for $j \in \mathbb{N}_{M / q}$ and a.e. $v \in$ $[0,1)$.

(iii) $\Psi_{\mathbf{g}}(j, v) \Psi_{\mathbf{g}}^{*}(j, v) \leq(B / M) I$ for $j \in \mathbb{Z}$ and a.e. $v \in \mathbb{R}$.

Proof. By Lemma 12, conditions (ii) and (iii) are equivalent. So, to finish the proof, we only need to prove that (i) holds if and only if

$$
\Psi_{\mathbf{g}}(j, v) \Psi_{\mathbf{g}}^{*}(j, v) \leq \frac{B}{M} I \quad \text { for } j \in \mathbb{N}_{M} \text {, a.e. } v \in[0,1),
$$

since $G(\mathbf{g}, \mathbf{N}, \mathbf{M})$ is a Bessel sequence with Bessel bound $B$ if and only if

$$
\left\|\mathscr{T}_{\mathbf{g}} c\right\|^{2} \leq B\|c\|^{2}
$$

for $c \in l_{0}\left(\mathbb{N}_{M} \times \mathbb{Z}, \mathbb{C}^{L}\right)$, which can be rewritten as

$$
\begin{aligned}
& \sum_{j \in \mathbb{N}_{M}} \int_{0}^{1}\left\|\overline{\Psi_{\mathbf{g}}^{*}(j, v)} \mathscr{J} c(j, v)\right\|^{2} d v \\
& \leq \frac{B}{M} \sum_{j \in \mathbb{N}_{M}} \int_{0}^{1}\|\mathscr{J} c(j, v)\|^{2} d v
\end{aligned}
$$

by Lemmas 6-10.

Next we prove the equivalence between (32) and (34) to finish the proof. It is obvious that (32) implies (34). Now we turn to the converse implication. Suppose (34) holds. Since every entry in $\Psi_{\mathbf{g}}(j, \cdot)$ belongs to $L^{2}([0,1))$ and thus belongs to $L^{1}([0,1))$ for $j \in \mathbb{N}_{M}$, almost every point in $(0,1)$ is a Lebesgue point for every entry in $\Psi_{\mathbf{g}}^{*}(j, v)$ and $j \in \mathbb{N}_{M}$. Let $v_{0}$ be such a point and $j_{0} \in \mathbb{N}_{M}$. Fix $x \in \mathbb{C}^{\mathrm{Q}}$ and $\epsilon>0$ with $\left(v_{0}-\epsilon, v_{0}+\epsilon\right) \subset(0,1)$. Define $c \in l^{2}\left(\mathbb{N}_{M} \times \mathbb{Z}, \mathbb{C}^{L}\right)$ by

$$
\mathscr{J} c(j, v)=\frac{1}{\sqrt{2 \epsilon}} \chi_{\left\{j_{0}\right\} \times\left(v_{0}-\epsilon, v_{0}+\epsilon\right)}(j, v) \bar{x}
$$

for $j \in \mathbb{N}_{M}, v \in[0,1)$. By Lemma 9 and the density of $l_{0}\left(\mathbb{N}_{M} \times\right.$ $\left.\mathbb{Z}, \mathbb{C}^{L}\right)$ in $l^{2}\left(\mathbb{N}_{M} \times \mathbb{Z}, \mathbb{C}^{L}\right),(34)$ holds for $c \in l^{2}\left(\mathbb{N}_{M} \times \mathbb{Z}, \mathbb{C}^{L}\right)$. Substituting (35) into (34), we obtain that

$$
\frac{1}{2 \epsilon} \int_{\left(v_{0}-\epsilon, v_{0}+\epsilon\right)}\left\|\Psi_{\mathbf{g}}^{*}\left(j_{0}, v\right) x\right\|^{2} d v \leq \frac{B}{M}\|x\|^{2} .
$$

Letting $\epsilon \rightarrow 0$ leads to

$$
\left\|\Psi_{\mathbf{g}}^{*}\left(j_{0}, v_{0}\right) x\right\|^{2} \leq \frac{B}{M}\|x\|^{2} ;
$$


that is,

$$
\left\langle\Psi_{\mathbf{g}}\left(j_{0}, v_{0}\right) \Psi_{\mathbf{g}}^{*}\left(j_{0}, v_{0}\right) x, x\right\rangle \leq \frac{\mathrm{B}}{M}\|x\|^{2} .
$$

This gives (32) by the arbitrariness of $j_{0}, v_{0}$, and $x$. The proof is completed.

Lemma 14 ([1, Theorem A.6.5]). Let $U_{1}, U_{2}, U_{3}$ be self-adjoint operators on a Hilbert space $\mathscr{H}$. If $U_{1} \leq U_{2}$, and $U_{3} \geq 0$, and $U_{3}$ commutes with $U_{1}$ and $U_{2}$, then $U_{1} U_{3} \leq U_{2} U_{3}$.

Remark 15. The inequality $\Psi_{\mathbf{g}}(j, v) \Psi_{\mathbf{g}}^{*}(j, v) \leq(B / M) I$ in Theorem 13 can be replaced by any one of the following inequalities:

(i) $\left(\Psi_{\mathbf{g}}(j, v) \Psi_{\mathbf{g}}^{*}(j, v)\right)^{2} \leq(B / M) \Psi_{\mathbf{g}}(j, v) \Psi_{\mathbf{g}}^{*}(j, v)$;

(ii) $\Psi_{\mathbf{g}}^{*}(j, v) \Psi_{\mathbf{g}}(j, v) \leq(B / M) I$;

(iii) $\left(\Psi_{\mathbf{g}}^{*}(j, v) \Psi_{\mathbf{g}}(j, v)\right)^{2} \leq(B / M) \Psi_{\mathbf{g}}^{*}(j, v) \Psi_{\mathbf{g}}(j, v)$.

Indeed, we have (i) by Lemma 14 if $\Psi_{\mathbf{g}}(j, v) \Psi_{\mathbf{g}}^{*}(j, v) \leq$ $(B / M) I$. From (i), we have

$$
\begin{gathered}
\left\langle\Psi_{\mathbf{g}}^{*}(j, v) \Psi_{\mathbf{g}}(j, v) \Psi_{\mathbf{g}}^{*}(j, v) x, \Psi_{\mathbf{g}}^{*}(j, v) x\right\rangle \\
\quad \leq \frac{B}{M}\left\langle\Psi_{\mathbf{g}}^{*}(j, v) x, \Psi_{\mathbf{g}}^{*}(j, v) x\right\rangle
\end{gathered}
$$

for $x \in \mathbb{C}^{Q}$, and thus $\Psi_{\mathbf{g}}^{*}(j, v) \Psi_{\mathbf{g}}(j, v) \leq(B / M) I$ on range $\left(\Psi_{\mathbf{g}}^{*}(j, v)\right)$. This implies (ii) due to the fact that $\mathbb{C}^{p}=$ $\operatorname{range}\left(\Psi_{\mathbf{g}}^{*}(j, v)\right) \oplus \operatorname{ker}\left(\Psi_{\mathbf{g}}(j, v)\right)$. It is obvious that (ii) implies (iii) by Lemma 14 . By the same procedure as in "(i) $\Rightarrow$ (ii)", we can prove that (iii) implies $\Psi_{\mathbf{g}}(j, v) \Psi_{\mathbf{g}}^{*}(j, v) \leq(B / M) I$.

Remark 16. Condition (iii) in Theorem 13 is equivalent to $\left\|\Psi_{\mathbf{g}}(j, v)\right\| \leq \sqrt{B / M}$ for a.e. $(j, v) \in \mathbb{Z} \times \mathbb{R}$ when $\Psi_{\mathbf{g}}(j, v)$ is understood as an operator from $\mathbb{C}^{p}$ into $\mathbb{C}^{\mathrm{Q}}$. However, such norm is equivalent to the one obtained by taking the maximum of the absolute values of all entries of a matrix. So $G(\mathbf{g}, \mathbf{N}, \mathbf{M})$ is a Bessel sequence if and only if $\mathscr{Z}_{q N} g_{l} \in$ $L^{\infty}(\mathbb{Z} \times \mathbb{R})$ for $1 \leq l \leq L$ by Theorem 13 .

Lemma 17. For $\mathbf{g}=\left(g_{1}, g_{2}, \ldots, g_{L}\right)$ with each $g_{l} \in l^{2}(\mathbb{Z})$, let $G(\mathbf{g}, \mathbf{N}, \mathbf{M})$ be a Bessel sequence in $l^{2}(\mathbb{Z})$. For $f \in l^{2}(\mathbb{Z})$, write $d^{(f)}=\mathscr{T}_{\mathbf{g}}^{*} f$. Then

$$
\mathscr{J} d^{(f)}(j, v)=M \overline{\Psi_{\mathbf{g}}(j, v)} \mathbf{Z}_{q N} f(j, v)
$$

for $(j, v) \in \mathbb{N}_{M} \times \mathbb{R}$ when either $G(\mathbf{g}, \mathbf{N}, \mathbf{M})$ is a Bessel sequence or $f \in l_{0}(\mathbb{Z})$.
Proof. By Lemmas 6 and 10, we have

$$
\begin{aligned}
& d_{l, m,(n q+r) \lambda_{l}+\tau_{l}}^{(f)} \\
& =\sum_{k \in \mathbb{N}_{p}} \sum_{j \in \mathbb{N}_{M}} \int_{0}^{1} \mathscr{Z}_{q N} f(j+k M, v) \\
& \quad \times \overline{\mathscr{Z}_{q N} g_{l}\left(j-\tau_{l} N_{l}-r N+k M, v\right)} \\
& \quad \times e^{-2 \pi i n v} d v e^{-2 \pi i(m / M) j} \\
& \sum_{j \in \mathbb{N}_{M}} \int_{0}^{1}\left(\overline{\mathscr{G}_{l}\left(j-\tau_{l} N_{l}, v\right)} \mathbf{Z}_{q N} f(j, v)\right)_{r} \\
& \times e^{-2 \pi i n v} d v e^{-2 \pi i(m / M) j}
\end{aligned}
$$

for each $r \in \mathbb{N}_{q}, \tau_{l} \in \mathbb{N}_{\lambda_{l}}$, and $1 \leq l \leq L$. When either $G(\mathbf{g}, \mathbf{N}, \mathbf{M})$ is a Bessel sequence or $f \in l_{0}(\mathbb{Z})$, the integrand in (41) belongs to $L^{2}([0,1))$ as a function about $v$ by Remark 16. It follows that

$$
\mathscr{D}^{\left(\tau_{l}\right)}(j, v)=M \overline{\mathscr{G}_{l}\left(j-\tau_{l} N_{l}, v\right)} \mathbf{Z}_{q N} f(j, v)
$$

for $(j, v) \in \mathbb{N}_{M} \times \mathbb{R}$. The lemma therefore follows.

Theorem 18. Let $G(\mathbf{g}, \mathbf{N}, \mathbf{M})$ and $G(\mathbf{h}, \mathbf{N}, \mathbf{M})$ be both Bessel sequences in $l^{2}(\mathbb{Z})$. Then

$$
\mathbf{Z}_{q N} \mathcal{S}_{\mathbf{h}, \mathbf{g}} f(j, v)=M \overline{\Psi_{\mathbf{g}}^{*}(j, v) \Psi_{\mathbf{h}}(j, v)} \mathbf{Z}_{q N} f(j, v)
$$

for $j \in \mathbb{N}_{M}$ and a.e. $v \in[0,1)$.

Proof. Write $d=\mathscr{T}_{\mathbf{h}}^{*} f$. Then

$$
\mathbf{Z}_{q N} \mathcal{\delta}_{\mathbf{h}, \mathbf{g}} f(j, v)=\overline{\Psi_{\mathbf{g}}^{*}(j, v)} \mathscr{J} d(j, v)
$$

by Remark 11. Applying Lemma 17 to $\mathbf{h}$, we have

$$
\mathscr{J} d(j, v)=M \bar{\Psi}_{\mathbf{h}}^{*}(j, v) \mathbf{Z}_{q N} f(j, v),
$$

and thus (43) holds by (44).

Theorem 19. For $\mathbf{g}=\left(g_{1}, g_{2}, \ldots, g_{L}\right)$ with each $g_{l} \in l^{2}(\mathbb{Z})$, the following are equivalent:

(i) $G(\mathbf{g}, \mathbf{N}, \mathbf{M})$ is a frame for $\mathscr{M}(\mathbf{g}, \mathbf{N}, \mathbf{M})$ with frame bounds $A$ and $B$;

(ii) $(A / M) \Psi_{\mathbf{g}}(j, v) \Psi_{\mathbf{g}}^{*}(j, v) \leq\left(\Psi_{\mathbf{g}}(j, v) \Psi_{\mathbf{g}}^{*}(j, v)\right)^{2} \leq(B /$ $M) \Psi_{\mathbf{g}}(j, v) \Psi_{\mathbf{g}}^{*}(j, v)$ for $j \in \mathbb{N}_{M / q}$ and a.e. $v \in[0,1)$;

(iii) $(A / M) \Psi_{\mathbf{g}}(j, v) \Psi_{\mathbf{g}}^{*}(j, v) \leq\left(\Psi_{\mathbf{g}}(j, v) \Psi_{\mathbf{g}}^{*}(j, v)\right)^{2} \leq(B /$ $M) \Psi_{\mathbf{g}}(j, v) \Psi_{\mathbf{g}}^{*}(j, v)$ for $j \in \mathbb{Z}$ and a.e. $v \in \mathbb{R}$.

Proof. By Theorem 13 and Remark 15, we may as well assume that $G(\mathbf{g}, \mathbf{N}, \mathbf{M})$ is a Bessel sequence, and we need to treat the "lower frame bound" part under this assumption. By a similar argument to beginning proof of Theorem 13, we only need to prove that

$$
\left\langle\mathcal{S}_{\mathbf{g}, \mathbf{g}} f, f\right\rangle \geq A\|f\|^{2} \quad \text { for } f \in \mathscr{M}(\mathbf{g}, \mathbf{N}, \mathbf{M})
$$


if and only if

$$
\begin{aligned}
& \left(\Psi_{\mathbf{g}}(j, v) \Psi_{\mathbf{g}}^{*}(j, v)\right)^{2} \\
& \quad \geq \frac{A}{M} \Psi_{\mathbf{g}}(j, v) \Psi_{\mathbf{g}}^{*}(j, v) \quad \text { for } j \in \mathbb{N}_{M}, v \in[0,1) .
\end{aligned}
$$

Since the linear span of $G(\mathbf{g}, \mathbf{N}, \mathbf{M})$ is dense in $\mathscr{M}(\mathbf{g}, \mathbf{N}, \mathbf{M})$, (46) holds if and only if

$$
\left\langle\mathcal{S}_{\mathbf{g}, \mathbf{g}} f, f\right\rangle \geq A\|f\|^{2}
$$

for $f=\mathscr{T}_{\mathrm{g}} c$ with $c \in l_{2}\left(\mathbb{N}_{M} \times \mathbb{Z}, \mathbb{C}^{L}\right)$ by [1, Lemma 5.1.7]. By Lemma 10, Theorem 18, and Remark 11, (48) can be rewritten as

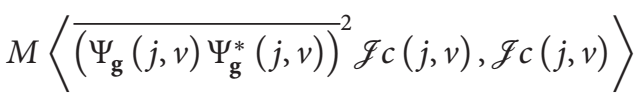

$$
\begin{aligned}
& \geq A\left\langle\overline{\Psi_{\mathbf{g}}(j, v) \Psi_{\mathbf{g}}^{*}(j, v)} \mathscr{J}_{c}(j, v), \mathscr{J}_{c}(j, v)\right\rangle,
\end{aligned}
$$

equivalently,

$$
\begin{aligned}
& M\left\langle\left(\Psi_{\mathbf{g}}(j, v) \Psi_{\mathbf{g}}^{*}(j, v)\right)^{2} \overline{\mathscr{J}_{c}(j, v)}, \overline{\mathscr{J}_{c}(j, v)}\right\rangle \\
& \quad \geq A\left\langle\Psi_{\mathbf{g}}(j, v) \Psi_{\mathbf{g}}^{*}(j, v) \overline{\mathscr{J}_{c}(j, v)}, \overline{\mathscr{J}_{c}(j, v)}\right\rangle
\end{aligned}
$$

for $j \in \mathbb{N}_{M}, v \in[0,1)$, and $c \in l^{2}\left(\mathbb{N}_{M} \times \mathbb{Z}, \mathbb{C}^{L}\right)$. By the same procedure as in Theorem 13, we can prove the equivalence between (47) and (50). The proof is completed.

Remark 20. By an argument similar to Remark 15, $\left(\Psi_{\mathbf{g}}(j, v) \Psi_{\mathbf{g}}^{*}(j, v)\right)^{2}$ and $\Psi_{\mathbf{g}}(j, v) \Psi_{\mathbf{g}}^{*}(j, v)$ in Theorem 19 can be replaced by $\left(\Psi_{\mathbf{g}}^{*}(j, v) \Psi_{\mathbf{g}}(j, v)\right)^{2}$ and $\Psi_{\mathbf{g}}^{*}(j, v) \Psi_{\mathbf{g}}(j, v)$, respectively.

Definition 21. Given $\mathbf{g}=\left(g_{1}, g_{2}, \ldots, g_{L}\right)$ with each $g_{l} \in l^{2}(\mathbb{Z})$, let $G(\mathbf{g}, \mathbf{N}, \mathbf{M})$ be a Bessel sequence in $l^{2}(\mathbb{Z})$. We say that $G(\mathbf{g}, \mathbf{N}, \mathbf{M})$ has Riesz property if, for $c \in l^{2}\left(\mathbb{N}_{M} \times \mathbb{Z}, \mathbb{C}^{L}\right)$, we must have $c=0$ whenever $\mathscr{T}_{\mathrm{g}} c=0$.

By an easy application of the spectral theorem of selfadjoint matrices, we have the following lemma (see also [36, page 978]).

Lemma 22. Given a measurable set $E$ in $\mathbb{R}$ with $|E|>0$, let $\mathscr{A}$ : $E \rightarrow M_{s, t}$ be a matrix-valued measurable function. Define by $\mathscr{P}(v)$ the orthogonal projection of $\mathbb{C}^{t}$ onto $\operatorname{ker}(\mathscr{A}(v))$. Then

$$
\mathscr{P}(v)=\lim _{n \rightarrow \infty} \exp \left(-n \mathscr{A}^{*}(v) \mathscr{A}(v)\right)
$$

for $v \in E$, and thus $\mathscr{P}(v)$ is measurable.

Theorem 23. For $\mathbf{g}=\left(g_{1}, g_{2}, \ldots, g_{L}\right)$ with each $g_{l} \in l^{2}(\mathbb{Z})$, the following are equivalent:

(i) $G(\mathbf{g}, \mathbf{N}, \mathbf{M})$ has Riesz property;

(ii) $\operatorname{rank}\left(\Psi_{\mathbf{g}}(j, v)\right)=Q$ for $j \in \mathbb{N}_{M / q}$ and a.e. $v \in[0,1)$;

(iii) $\operatorname{rank}\left(\Psi_{\mathbf{g}}(j, v)\right)=Q$ for $j \in \mathbb{Z}$ and a.e. $v \in \mathbb{R}$.
Proof. By Lemma 12, (ii) and (iii) are equivalent. So we only need to prove that $c=0$ is a unique solution to

$$
\mathscr{T}_{\mathbf{g}} c=0
$$

in $l^{2}\left(\mathbb{N}_{M} \times \mathbb{Z}, \mathbb{C}^{L}\right)$ if and only if

$$
\operatorname{rank}\left(\Psi_{\mathbf{g}}(j, v)\right)=Q \quad \text { for } j \in \mathbb{N}_{M}, v \in[0,1) .
$$

By Remark 11, (52) can be rewritten as

$$
\overline{\Psi_{\mathrm{g}}^{*}(j, v)} \mathscr{L}_{c}(j, v)=0 \quad \text { for } j \in \mathbb{N}_{M}, v \in[0,1) .
$$

So $c=0$ is a unique solution to (52) in $l^{2}\left(\mathbb{N}_{M} \times \mathbb{Z}, \mathbb{C}^{L}\right)$ if and only if $d(j, v)=0$ is a unique solution to

$$
\overline{\Psi_{\mathbf{g}}^{*}(j, v)} d(j, v)=0 \quad \text { for } j \in \mathbb{N}_{M} \text {, a.e. } v \in[0,1)
$$

in $L^{2}\left(\mathbb{N}_{M} \times[0,1), \mathbb{C}^{\mathrm{Q}}\right)$ by Lemma 9 . It is obvious that (53) implies that $d(j, v)=0$ is a unique solution to (55) in $L^{2}\left(\mathbb{N}_{M} \times\right.$ $\left.[0,1), \mathbb{C}^{\mathrm{Q}}\right)$. Next we prove the converse implication. Suppose there exist $j_{0} \in \mathbb{N}_{M}$ and $E \subset[0,1)$ with $|E|>0$ such that $\operatorname{rank}\left(\Psi_{\mathbf{g}}\left(j_{0}, v\right)\right)<Q$ on $E$. Let $\mathscr{P}\left(j_{0}, v\right)$ be the orthogonal projection of $\mathbb{C}^{\mathrm{Q}}$ onto $\operatorname{ker}\left(\Psi_{\mathrm{g}}^{*}\left(j_{0}, v\right)\right)$. Then there exist $i_{0} \in \mathbb{N}_{\mathrm{Q}}$ and $E^{\prime} \subset E$ with $\left|E^{\prime}\right|>0$ such that $\mathscr{P}\left(j_{0}, v\right) \mathbf{e}_{i_{0}} \neq 0$ for $v \in E^{\prime}$. Define $d(j, v) \in L^{2}\left(\mathbb{N}_{M} \times[0,1), \mathbb{C}^{\mathrm{Q}}\right)$ by

$$
d(j, v)= \begin{cases}\mathscr{P}(j, v) \mathbf{e}_{i_{0}}, & \text { if } j=j_{0}, v \in E^{\prime} \\ 0, & \text { otherwise }\end{cases}
$$

for $j \in \mathbb{N}_{M}$ and $v \in[0,1)$. Then $d(j, v)$ is well defined, and $\|d(j, v)\| \leq 1$ by Lemma 22 . It follows that $d(j, v)$ is a nonzero solution to (55) in $L^{2}\left(\mathbb{N}_{M} \times[0,1), \mathbb{C}^{\mathrm{Q}}\right)$. This is a contradiction. The proof is completed.

Since a Riesz basis is exactly a frame having Riesz property, and an orthonormal basis is exactly a Riesz basis with Riesz bound 1, we have the following theorem by Theorems 19 and 23.

Theorem 24. For $\mathbf{g}=\left(g_{1}, g_{2}, \ldots, g_{L}\right)$ with each $g_{l} \in l^{2}(\mathbb{Z})$, the following are equivalent:

(i) $G(\mathbf{g}, \mathbf{N}, \mathbf{M})$ is a Riesz basis for $G(\mathbf{g}, \mathbf{N}, \mathbf{M})$ with Riesz bounds $A$ and $B$ (an orthonormal basis);

(ii) $(A / M) I \leq \Psi_{\mathbf{g}}(j, v) \Psi_{\mathbf{g}}^{*}(j, v) \leq(B / M) I\left(\Psi_{\mathbf{g}}(j, v) \Psi_{\mathbf{g}}^{*}(j\right.$, $v)=(1 / M) I)$ for $j \in \mathbb{N}_{M / q}$ a.e. $v \in[0,1) ;$

(iii) $(A / M) I \leq \Psi_{\mathbf{g}}(j, v) \Psi_{\mathbf{g}}^{*}(j, v) \leq(B / M) I\left(\Psi_{\mathbf{g}}(j, v) \Psi_{\mathbf{g}}^{*}(j\right.$, $v)=(1 / M) I)$ for $j \in \mathbb{Z}$ and a.e. $v \in \mathbb{R}$.

Next we turn to examples of Theorems 19 and 24. Suppose

$$
\mathbf{N}=(6,4), \quad \mathbf{M}=(2,2) .
$$


Then $N / M=6$ with $p=6, q=1$, and $Q=5$. For $\mathbf{g}=\left(g_{1}, g_{2}\right)$ with $g_{1}, g_{2} \in l^{2}(\mathbb{Z})$, we associate it with $\Psi_{\mathrm{g}}$ as in Definition 4 . Then

$\Psi_{\mathbf{g}}(j, v)=\left(\begin{array}{l}G_{1}(j, v) \\ G_{2}(j, v)\end{array}\right)$ with

$G_{1}(j, v)=\left(\begin{array}{c}\mathscr{G}_{1}(j, v) \\ \mathscr{G}_{1}(j-6, v)\end{array}\right), \quad G_{2}(j, v)=\left(\begin{array}{c}\mathscr{G}_{2}(j, v) \\ \mathscr{G}_{2}(j-4, v) \\ \mathscr{G}_{2}(j-8, v)\end{array}\right)$,

where

$$
\begin{array}{r}
\mathscr{G}_{l}(j, v)=\left(\mathscr{Z}_{12} g_{l}(j+2 k, v)\right)_{0, k} \in \mathscr{M}_{1,6} \\
\text { with } j \in \mathbb{N}_{2}, k \in \mathbb{N}_{6}
\end{array}
$$

for $l=1,2$. By the quasi-periodicity of Zak transform, we have

$$
\begin{aligned}
\mathscr{G}_{1} & (j-6, v) \\
= & \left(e^{2 \pi i v} \mathscr{E}_{12} g_{1}(j+6, v), e^{2 \pi i v} \mathscr{Z}_{12} g_{1}(j+8, v),\right. \\
& e^{2 \pi i v} \mathscr{Z}_{12} g_{1}(j+10, v), \mathscr{E}_{12} g_{1}(j, v), \\
& \left.\mathscr{Z}_{12} g_{1}(j+2, v), \mathscr{Z}_{12} g_{1}(j+4, v)\right),
\end{aligned}
$$

$$
\begin{gathered}
\mathscr{G}_{2}(j-4, v) \\
=\left(e^{2 \pi i v} \mathscr{E}_{12} g_{2}(j+8, v), e^{2 \pi i v} \mathscr{Z}_{12} g_{2}(j+10, v),\right. \\
\mathscr{Z}_{12} g_{2}(j, v), \mathscr{E}_{12} g_{2}(j+2, v), \\
\left.\mathscr{E}_{12} g_{2}(j+4, v), \mathscr{E}_{12} g_{2}(j+6, v)\right), \\
\mathscr{G}_{2}(j-8, v) \\
=\left(e^{2 \pi i v} \mathscr{Z}_{12} g_{2}(j+4, v), e^{2 \pi i v} \mathscr{Z}_{12} g_{2}(j+6, v),\right. \\
e^{2 \pi i v} \mathscr{Z}_{12} g_{2}(j+8, v), \mathscr{Z}_{12} g_{2}(j+10, v), \\
\left.\mathscr{Z}_{12} g_{2}(j, v), \mathscr{Z}_{12} g_{2}(j+2, v)\right) .
\end{gathered}
$$

Thus for each $j \in \mathbb{N}_{2}$ and a.e. $v \in[0,1), \mathscr{G}_{1}(j-6, v)$, and $\mathscr{G}_{2}(j-4, v), \mathscr{G}_{2}(j-8, v)$ are uniquely determined by $\mathscr{G}_{1}(j, v)$ and $\mathscr{G}_{2}(j, v)$, respectively. Observe that $\mathbb{N}_{2}+2 \mathbb{N}_{6}$ is $12 \mathbb{Z}$ congruent to $\mathbb{N}_{12}$. It follows that $\mathrm{g}$ is uniquely determined by the values of $\Psi_{\mathbf{g}}(j, v)$ for $j \in \mathbb{N}_{2}$ and a.e. $v \in[0,1)$. Therefore, an arbitrary matrix $5 \times 6$ matrix-valued function $\mathscr{K}(j, v)$ for $j \in \mathbb{N}_{2}$ and a.e. $v \in[0,1)$ with all entries being in $L^{2}\left(\mathbb{N}_{2} \times[0,1)\right)$ determines a unique $\mathbf{g}$ by

$$
\Psi_{\mathbf{g}}(j, v)=\mathscr{K}(j, v) \quad \text { for } j \in \mathbb{N}_{2} \text {, a.e. } v \in[0,1) .
$$

Let

$$
\begin{aligned}
& \mathscr{K}(j, v) \\
& =\left(\begin{array}{cccccc}
a(j, v) & \lambda(j, v) a(j, v) & \lambda^{2}(j, v) a(j, v) & 0 & 0 & 0 \\
0 & 0 & 0 & a(j, v) & \lambda(j, v) a(j, v) & \lambda^{2}(j, v) a(j, v) \\
-\overline{\lambda(j, v) a(j, v)} & \overline{a(j, v)} & 0 & 0 & 0 & 0 \\
0 & 0 & \frac{\lambda}{\lambda(j, v) a(j, v)} & \frac{a(j, v)}{a(j, v)}
\end{array}\right)
\end{aligned}
$$

for $j \in \mathbb{N}_{2}$ and a.e. $v \in[0,1)$. Define $\mathbf{g}=\left(g_{1}, g_{2}\right)$ by

$$
\Psi_{\mathbf{g}}(j, v)=\mathscr{K}(j, v) \quad \text { for } j \in \mathbb{N}_{2} \text {, a.e. } v \in[0,1] .
$$

Then we obtain the following example.

Example 25. Let $\mathbf{N}$ and $\mathbf{M}$ be defined as in (57) and define $\mathbf{g}=\left(g_{1}, g_{2}\right)$ by $(63)$, where $\lambda(j, \cdot), a(j, \cdot)$ are continuous on $[0,1]$ for $j \in\{0,1\}$. Assume that

$$
\begin{gathered}
|\lambda(j, v)|<1, \\
|a(j, v)|^{2}\left(1-2|\lambda(j, v)|^{4}-2|\lambda(j, v)|^{6}-|\lambda(j, v)|^{8}\right) \\
<1-|\lambda(j, v)|^{3},
\end{gathered}
$$

$$
\begin{aligned}
& |a(j, v)|^{2}\left(1+2|\lambda(j, v)|^{3}+2|\lambda(j, v)|^{5}+|\lambda(j, v)|^{7}\right) \\
& <1+|\lambda(j, v)|^{3}
\end{aligned}
$$

for $(j, v) \in\{0,1\} \times[0,1]$ satisfying $a(j, v) \neq 0$. Then

$$
\operatorname{supp}\left(g_{1}\right) \subset \mathbb{N}_{6}+12 \mathbb{Z}, \quad \operatorname{supp}\left(g_{2}\right) \subset \mathbb{N}_{4}+12 \mathbb{Z},
$$

and $G(\mathbf{g}, \mathbf{N}, \mathbf{M})$ is a frame for $\mathscr{M}(\mathbf{g}, \mathbf{N}, \mathbf{M})$. 
Proof. Equation (65) is an immediate consequence of (63). Next we prove that $G(\mathbf{g}, \mathbf{N}, \mathbf{M})$ is a frame for $\mathscr{M}(\mathbf{g}, \mathbf{N}, \mathbf{M})$. By a simple computation, we have

$$
\begin{aligned}
& \left\langle\left(\Psi_{\mathbf{g}}(j, v) \Psi_{\mathbf{g}}^{*}(j, v)\right)^{2} x, x\right\rangle \\
& \quad=|a(j, v)|^{2} \sum_{l=1}^{5} \alpha_{l}(j, v)\left|x_{l}\right|^{2}+\beta(j, v), \\
& \left\langle\Psi_{\mathbf{g}}(j, v) \Psi_{\mathbf{g}}^{*}(j, v) x, x\right\rangle \\
& \quad=|a(j, v)|^{2} \sum_{l=1}^{5} \widetilde{\alpha}_{l}(j, v)\left|x_{l}\right|^{2}+\widetilde{\beta}(j, v)
\end{aligned}
$$

for $(j, v) \in\{0,1\} \times[0,1]$ and $x \in \mathbb{C}^{5}$, where

$$
\begin{aligned}
\alpha_{1}(j, v)= & |\lambda(j, v)|^{3} \\
& +\left(1+|\lambda(j, v)|^{2}+|\lambda(j, v)|^{4}\right)^{2}|a(j, v)|^{2}, \\
\alpha_{2}(j, v)= & +\left(1+|\lambda(j, v)|^{2}+|\lambda(j, v)|^{4}\right)^{2} \\
& \times|a(j, v)|^{2}, \\
\alpha_{4}(j, v)= & 1+|\lambda(j, v)|^{3}+\left(1+|\lambda(j, v)|^{2}\right)^{2}|a(j, v)|^{2}, \\
\alpha_{4}(j, v)= & 1+|\lambda(j, v)|^{3}+\left(1+|\lambda(j, v)|^{2}\right)^{2}|a(j, v)|^{2}, \\
\alpha_{3}(j, v)= & \alpha_{5}(j, v)=\left(1+|\lambda(j, v)|^{2}\right)^{2}|a(j, v)|^{2}, \\
\beta(j, v)= & 2 \operatorname{Re}\left(\left[\lambda(j, v)^{3}\right.\right. \\
& \times\left(2+2|\lambda(j, v)|^{2}+|\lambda(j, v)|^{4}\right) \\
& \left.\left.\quad \times a(j, v)^{2}|a(j, v)|^{2}\right] \bar{x}_{1} x_{4}\right) \\
& +2 \operatorname{Re}\left(\left[\left(2+2|\lambda(j, v)|^{2}+|\lambda(j, v)|^{4}\right)\right.\right. \\
& \left.\left.\quad \times a(j, v)^{2}|a(j, v)|^{2}\right] \bar{x}_{2} x_{4}\right) \\
& +2 \operatorname{Re}\left(\lambda(j, v)^{3}|a(j, v)|^{2} \bar{x}_{1} x_{2}\right), \\
\widetilde{\alpha}_{1}(j, v)= & \widetilde{\alpha}_{2}(j, v)=1+|\lambda(j, v)|^{2}+|\lambda(j, v)|^{4}, \\
\widetilde{\alpha}_{3}(j, v)= & \widetilde{\alpha}_{4}(j, v)=\widetilde{\alpha}_{5}(j, v)=1+|\lambda(j, v)|^{2}, \\
\widetilde{\beta}(j, v)= & -2 \operatorname{Re}\left(\lambda(j, v)^{3} a(j, v)^{2} \bar{x}_{1} x_{4}\right) \\
& \left.+2 \operatorname{Re} \bar{x}_{2} x_{4}\right) .
\end{aligned}
$$

Write

$$
\begin{gathered}
\widetilde{\beta}_{1}(j, v)=|\lambda(j, v)|^{3}, \quad \widetilde{\beta}_{2}(j, v)=1, \\
\widetilde{\beta}_{4}(j, v)=1+|\lambda(j, v)|^{3} .
\end{gathered}
$$

where

$$
\begin{gathered}
C_{l}(j, v)=\alpha_{l}(j, v)+\beta_{l}(j, v), \\
\widetilde{C}_{l}(j, v)=\alpha_{l}(j, v)-\beta_{l}(j, v) \quad \text { for } l=1,2,4, \\
C(j, v)=\left(1+|\lambda(j, v)|^{2}\right)^{2}|a(j, v)|^{2}, \\
D_{l}(j, v)=\widetilde{\alpha}_{l}(j, v)+\widetilde{\beta}_{l}(j, v), \\
\widetilde{D}_{l}(j, v)=\widetilde{\alpha}_{l}(j, v)-\widetilde{\beta}_{l}(j, v) \quad \text { for } l=1,2,4, \\
D(j, v)=|\lambda(j, v)|^{2}+1 .
\end{gathered}
$$

Take

$$
\begin{aligned}
& \widetilde{C}=\min \left\{C(j, v), \widetilde{C}_{l}(j, v): l=1,2,4, j \in \mathbb{N}_{2}, v \in[0,1]\right\}, \\
& C=\max \left\{C(j, v), C_{l}(j, v): l=1,2,4, j \in \mathbb{N}_{2}, v \in[0,1]\right\}, \\
& \widetilde{D}=\min \left\{D(j, v), \widetilde{D}_{l}(j, v): l=1,2,4, j \in \mathbb{N}_{2}, v \in[0,1]\right\}, \\
& D=\max \left\{D(j, v), D_{l}(j, v): l=1,2,4, j \in \mathbb{N}_{2}, v \in[0,1]\right\} .
\end{aligned}
$$$$
|\beta(j, v)| \leq|a(j, v)|^{2}\left[\beta_{1}(j, v)\left|x_{1}\right|^{2}+\beta_{2}(j, v)\left|x_{2}\right|^{2}\right.
$$$$
\left.+\beta_{4}(j, v)\left|x_{4}\right|^{2}\right],
$$ 
Then

$$
\begin{aligned}
& \left\langle\left(\Psi_{\mathbf{g}}(j, v) \Psi_{\mathbf{g}}^{*}(j, v)\right)^{2} x, x\right\rangle \\
& \leq|a(j, v)|^{2}\left[C_{1}(j, v)\left|x_{1}\right|^{2}+C_{2}(j, v)\left|x_{2}\right|^{2}\right. \\
& +C_{4}(j, v)\left|x_{4}\right|^{2}+C(j, v) \\
& \left.\times\left(\left|x_{3}\right|^{2}+\left|x_{5}\right|^{2}\right)\right] \\
& \leq C|a(j, v)|^{2}\|x\|^{2} \text {, } \\
& \left\langle\left(\Psi_{\mathbf{g}}(j, v) \Psi_{\mathbf{g}}^{*}(j, v)\right)^{2} x, x\right\rangle \\
& \geq|a(j, v)|^{2}\left[\widetilde{C}_{1}(j, v)\left|x_{1}\right|^{2}+\widetilde{C}_{2}(j, v)\left|x_{2}\right|^{2}\right. \\
& +\widetilde{C}_{4}(j, v)\left|x_{4}\right|^{2}+C(j, v) \\
& \left.\times\left(\left|x_{3}\right|^{2}+\left|x_{5}\right|^{2}\right)\right] \\
& \geq \widetilde{C}|a(j, v)|^{2}\|x\|^{2}, \\
& \left\langle\Psi_{\mathbf{g}}(j, v) \Psi_{\mathbf{g}}^{*}(j, v) x, x\right\rangle \\
& \leq|a(j, v)|^{2}\left[D_{1}(j, v)\left|x_{1}\right|^{2}+D_{2}(j, v)\left|x_{2}\right|^{2}\right. \\
& +D_{4}(j, v)\left|x_{4}\right|^{2}+D(j, v) \\
& \left.\times\left(\left|x_{3}\right|^{2}+\left|x_{5}\right|^{2}\right)\right] \\
& \leq D|a(j, v)|^{2}\|x\|^{2}, \\
& \left\langle\Psi_{\mathbf{g}}(j, v) \Psi_{\mathbf{g}}^{*}(j, v) x, x\right\rangle \\
& \geq|a(j, v)|^{2}\left[\widetilde{D}_{1}(j, v)\left|x_{1}\right|^{2}\right. \\
& +\widetilde{D}_{2}(j, v)\left|x_{2}\right|^{2}+\widetilde{D}_{4}(j, v)\left|x_{4}\right|^{2} \\
& \left.+D(j, v)\left(\left|x_{3}\right|^{2}+\left|x_{5}\right|^{2}\right)\right] \\
& \geq \widetilde{D}|a(j, v)|^{2}\|x\|^{2} .
\end{aligned}
$$

It follows that

$$
\begin{aligned}
\frac{A}{2}\left\langle\Psi_{\mathbf{g}}(j, v) \Psi_{\mathbf{g}}^{*}(j, v) x, x\right\rangle & \leq\left\langle\left(\Psi_{\mathbf{g}}(j, v) \Psi_{\mathbf{g}}^{*}(j, v)\right)^{2} x, x\right\rangle \\
& \leq \frac{B}{2}\left\langle\Psi_{\mathbf{g}}(j, v) \Psi_{\mathbf{g}}^{*}(j, v) x, x\right\rangle,
\end{aligned}
$$

for $x \in \mathbb{C}^{5}, j \in \mathbb{N}_{2}$, and a.e. $v \in[0,1)$, and thus

$$
\begin{aligned}
\frac{A}{2} \Psi_{\mathbf{g}}(j, v) \Psi_{\mathbf{g}}^{*}(j, v) & \leq\left(\Psi_{\mathbf{g}}(j, v) \Psi_{\mathbf{g}}^{*}(j, v)\right)^{2} \\
& \leq \frac{B}{2} \Psi_{\mathbf{g}}(j, v) \Psi_{\mathbf{g}}^{*}(j, v)
\end{aligned}
$$

for $j \in \mathbb{N}_{2}$ and a.e. $v \in[0,1)$, where $A=2 \widetilde{C} / D, B=2 C / \widetilde{D}$. By Theorem $19, G(\mathbf{g}, \mathbf{N}, \mathbf{M})$ is a frame for $\mathscr{M}(\mathbf{g}, \mathbf{N}, \mathbf{M})$ with frame bounds $A$ and $B$.
Remark 26. By a simple computation, we have $\operatorname{rank}\left(\Psi_{\mathbf{g}}(j\right.$, $v))=5$ if and only if

$$
a(0, v) a(1, v) \lambda(0, v) \lambda(1, v) \neq 0 \quad \text { for a.e. } v \in[0,1) .
$$

Also observe that $\operatorname{rank}\left(\Psi_{\mathbf{g}}(j, v)\right)=5$ is equivalent to $\Psi_{\mathbf{g}}(j, v) \Psi_{\mathbf{g}}^{*}(j, v)$ being invertible, and that (75) can be reduced to

$$
\frac{A I}{2} \leq \Psi_{\mathbf{g}}(j, v) \Psi_{\mathbf{g}}^{*}(j, v) \leq \frac{B I}{2} \quad \text { for } j \in \mathbb{N}_{2} \text {, a.e. } v \in[0,1)
$$

in this case. Therefore, $G(\mathbf{g}, \mathbf{N}, \mathbf{M})$ is a Riesz basis for $\mathscr{M}(\mathbf{g}, \mathbf{N}, \mathbf{M})$ with Riesz bounds $A$ and $B$ if and only if (76) holds.

\section{Gabor Dual Characterization}

Let $G(\mathbf{g}, \mathbf{N}, \mathbf{M})$ be a frame for $\mathscr{M}(\mathbf{g}, \mathbf{N}, \mathbf{M})$. In this section, we discuss three kinds of duals with Gabor structure. We establish characterizations of Gabor duals of type I and type II and obtain a sufficient condition for Gabor duals of type II and oblique Gabor duals.

By an argument similar to [5, Lemma 2.6], we have the following lemmas.

Lemma 27. For $\mathbf{g}=\left(g_{1}, g_{2}, \ldots, g_{L}\right)$ and $\mathbf{h}=\left(h_{1}, h_{2}, \ldots, h_{L}\right)$ with each $g_{l}, h_{l} \in l^{2}(\mathbb{Z})$, the following are equivalent:

(i) $h_{l} \in \mathscr{M}(\mathbf{g}, \mathbf{N}, \mathbf{M})$ for each $1 \leq l \leq L$;

(ii) there exists a function $A: \mathbb{N}_{M / q} \times[0,1) \rightarrow \mathscr{M}_{\mathrm{Q}, \mathrm{Q}}$ such that

$$
\Psi_{\mathbf{h}}(j, v)=A(j, v) \Psi_{\mathbf{g}}(j, v)
$$

for $j \in \mathbb{N}_{M / q}$ and a.e. $v \in[0,1)$;

(iii) there exists a function $A: \mathbb{Z} \times \mathbb{R} \rightarrow \mathscr{M}_{\mathrm{Q}, \mathrm{Q}}$ such that

$$
\Psi_{\mathbf{h}}(j, v)=A(j, v) \Psi_{\mathbf{g}}(j, v)
$$

for $j \in \mathbb{Z}$ and a.e. $v \in \mathbb{R}$.

Lemma 28. Given $\mathbf{g}=\left(g_{1}, g_{2}, \ldots, g_{L}\right)$ with each $g_{l} \in l^{2}(\mathbb{Z})$, $\mathbf{h}=\left(h_{1}, h_{2}, \ldots, h_{L}\right)$ with each $h_{l} \in l^{2}(\mathbb{Z})$, let $G(\mathbf{g}, \mathbf{N}, \mathbf{M})$ and $G(\mathbf{h}, \mathbf{N}, \mathbf{M})$ be both Bessel sequences in $l^{2}(\mathbb{Z})$. Then the following are equivalent:

(i) $\operatorname{range}\left(\mathscr{T}_{\mathbf{h}}^{*}\right) \subset \overline{\operatorname{range}\left(\mathscr{T}_{\mathrm{g}}^{*}\right)}$;

(ii) there exists a function $B: \mathbb{N}_{M / q} \times[0,1) \rightarrow \mathscr{M}_{p, p}$ such that

$$
\Psi_{\mathbf{h}}(j, v)=\Psi_{\mathbf{g}}(j, v) B(j, v) \quad \text { for }(j, v) \in \mathbb{N}_{M / q} \times[0,1) ;
$$

(iii) there exists a function $B: \mathbb{Z} \times \mathbb{R} \rightarrow \mathscr{M}_{p, p}$ such that

$$
\Psi_{\mathbf{h}}(j, v)=\Psi_{\mathbf{g}}(j, v) B(j, v) \quad \text { for }(j, v) \in \mathbb{Z} \times \mathbb{R} .
$$


Proof. If (ii) holds, we can obtain (iii) by choosing $B: \mathbb{Z} \times$ $\mathbb{R} \rightarrow \mathscr{M}_{p, p}$ as

$$
B\left(j+\frac{M}{q} n, v\right)=\mathscr{D}_{n}^{*}(v) B(j, v) \mathscr{D}_{n}(v)
$$

for $(j, v) \in \mathbb{N}_{M / q} \times \mathbb{R}$, where $\mathscr{D}_{n}(v)$ is defined as in Lemma 12 . So we only need to prove that (i) holds if and only if there exists a function $B: \mathbb{N}_{M} \times[0,1) \rightarrow \mathscr{M}_{p, p}$ such that

$$
\Psi_{\mathbf{h}}(j, v)=\Psi_{\mathbf{g}}(j, v) B(j, v) \quad \text { for }(j, v) \in \mathbb{N}_{M} \times[0,1) .
$$

For $c \in l^{2}\left(\mathbb{N}_{M} \times \mathbb{Z}, \mathbb{C}^{L}\right), c$ is orthogonal to $\operatorname{range}\left(\mathscr{T}_{\mathrm{g}}^{*}\right)$ $\left(\operatorname{range}\left(\mathscr{T}_{\mathbf{h}}^{*}\right)\right)$ if and only if

$$
\begin{gathered}
\mathscr{J}_{c}(j, v) \perp \overline{\Psi_{\mathbf{g}}(j, v)} \mathbf{Z}_{q N} f(j, v) \\
\left(\mathscr{J}_{c}(j, v) \perp \overline{\Psi_{\mathbf{h}}(j, v)} \mathbf{Z}_{q N} f(j, v)\right)
\end{gathered}
$$

in $L^{2}\left(\mathbb{N}_{M} \times[0,1), \mathbb{C}^{\mathrm{Q}}\right)$-inner product by Lemmas 9 and 17 , equivalently,

$$
\begin{gathered}
\overline{\Psi_{\mathrm{g}}^{*}(j, v)} \mathscr{\mathscr { J }} c(j, v) \perp \mathbf{Z}_{q N} f(j, v) \\
\left(\overline{\Psi_{\mathbf{h}}^{*}(j, v)} \mathscr{J} c(j, v) \perp \mathbf{Z}_{q N} f(j, v)\right) \quad \text { for } f \in l^{2}(\mathbb{Z})
\end{gathered}
$$

in $L^{2}\left(\mathbb{N}_{M} \times[0,1), \mathbb{C}^{p}\right)$-inner. Again by Lemma $6,(85)$ is equivalent to

$$
\overline{\Psi_{\mathbf{g}}^{*}(j, v)} \mathscr{J}_{c}(j, v)=0 \quad\left(\overline{\Psi_{\mathbf{h}}^{*}(\mathrm{j}, v)} \mathscr{J} c(j, v)=0\right)
$$$$
\text { for } j \in \mathbb{N}_{M} \text {, a.e. } v \in[0,1) \text {. }
$$

So we only need to prove that (83) holds if and only if, for $\mathscr{J} c(j, v) \in L^{2}\left(\mathbb{N}_{M} \times[0,1), \mathbb{C}^{\mathrm{Q}}\right)$,

$$
\overline{\Psi_{\mathbf{h}}^{*}(j, v)} \mathscr{J} c(j, v)=0 \quad \text { for } j \in \mathbb{N}_{M} \text {, a.e. } v \in[0,1)
$$

whenever

$$
\overline{\Psi_{\mathrm{g}}^{*}(j, v)} \mathscr{F} c(j, v)=0 \text { for } j \in \mathbb{N}_{M} \text {, a.e. } v \in[0,1) .
$$

Obviously the necessity holds. Next we prove that a contradiction will occur if (83) is violated. Suppose (83) does not hold. Then there exist some $j_{0} \in \mathbb{N}_{M}$ and $E \subset[0,1)$ with $|E|>0$, on which some $r_{0}$ th column $h_{r_{0}}$ of $\overline{\Psi_{\mathbf{h}}\left(j_{0}, v\right)}$ satisfies

$$
h_{r_{0}} \notin \operatorname{range}\left(\overline{\Psi_{\mathbf{g}}\left(j_{0}, v\right)}\right) \quad \text { for } v \in E .
$$

Let $\mathscr{P}\left(j_{0}, v\right)$ be the orthogonal projection of $\mathbb{C}^{\mathrm{Q}}$ onto $\operatorname{ker}\left(\overline{\Psi_{\mathbf{g}}^{*}\left(j_{0}, v\right)}\right)$. Define $\mathscr{J} c(j, v) \in L^{2}\left(\mathbb{N}_{M} \times[0,1), \mathbb{C}^{\mathrm{Q}}\right)$ by

$$
\mathscr{J}_{c}(j, v)= \begin{cases}\mathscr{P}\left(j_{0}, v\right) h_{r_{0}}\left(j_{0}, v\right), & \text { if } j=j_{0}, v \in E \\ 0, & \text { otherwise }\end{cases}
$$

for $(j, v) \in \mathbb{N}_{M} \times[0,1)$. Then $\mathscr{J} c(j, v)$ solves (88) and $\mathscr{F}_{c}\left(j_{0}, v\right) \neq 0$ for $v \in E$. Also observe that the $r_{0}$ th component of $\overline{\Psi_{\mathbf{h}}^{*}\left(j_{0}, v\right)} \mathscr{F} c\left(j_{0}, v\right)$ is exactly $\left\|\mathscr{J} c\left(j_{0}, v\right)\right\|^{2}$ for $v \in E$. It follows that $\mathscr{J}_{c}(j, v)$ fails to solve (87). This finishes the proof.
Lemma 29. Given $\mathbf{g}=\left(g_{1}, g_{2}, \ldots, g_{L}\right), \mathbf{h}=\left(h_{1}, h_{2}, \ldots, h_{L}\right)$ with each $g_{l}, h_{l} \in l^{2}(\mathbb{Z})$, let $G(\mathbf{g}, \mathbf{N}, \mathbf{M})$ and $G(\mathbf{h}, \mathbf{N}, \mathbf{M})$ be both Bessel sequences in $l^{2}(\mathbb{Z})$. Then

$$
\mathcal{S}_{\mathbf{h}, \mathbf{g}} f=f
$$

for $f \in \mathscr{M}(\mathbf{g}, \mathbf{N}, \mathbf{M})$ if and only if

$$
\Psi_{\mathbf{g}}^{*}(j, v)=M \Psi_{\mathbf{g}}^{*}(j, v) \Psi_{\mathbf{h}}(j, v) \Psi_{\mathbf{g}}^{*}(j, v)
$$

for $j \in \mathbb{N}_{M / q}$ and a.e. $v \in[0,1)$.

Proof. By Lemma 12, (92) holds for $j \in \mathbb{N}_{M / q}$ and a.e. $v \in$ $[0,1)$ if and only if it holds for $j \in \mathbb{N}_{M}$ and a.e. $v \in[0,1)$. Next we prove that (91) holds for $f \in \mathscr{M}(\mathbf{g}, \mathbf{N}, \mathbf{M})$ if and only if (92) holds for $j \in \mathbb{N}_{M}$ and a.e. $v \in[0,1)$.

Since range $\left(\mathscr{T}_{\mathbf{g}}\right)$ is dense in $\mathscr{M}(\mathbf{g}, \mathbf{N}, \mathbf{M})$, (91) holds for $f \in \mathscr{M}(\mathbf{g}, \mathbf{N}, \mathbf{M})$ if and only if it holds for $f \in \operatorname{range}\left(\mathscr{T}_{\mathbf{g}}\right)$, equivalently,

$$
\begin{aligned}
& \Psi_{\mathbf{g}}^{*}(j, v) \mathscr{J}_{c}(j, v) \\
& \quad=M \Psi_{\mathbf{g}}^{*}(j, v) \Psi_{\mathbf{h}}(j, v) \Psi_{\mathbf{g}}^{*}(j, v) \mathscr{J}_{c}(j, v)
\end{aligned}
$$

for $c \in l^{2}\left(\mathbb{N}_{M} \times \mathbb{Z}, \mathbb{C}^{L}\right)$ by Remark 11 and Theorem 18 . This is also equivalent to

$$
\begin{aligned}
& \Psi_{\mathbf{g}}^{*}(j, v) d(j, v) \\
& \quad=M \Psi_{\mathbf{g}}^{*}(j, v) \Psi_{\mathbf{h}}(j, v) \Psi_{\mathbf{g}}^{*}(j, v) d(j, v)
\end{aligned}
$$

for $d(j, v) \in L^{2}\left(\mathbb{N}_{M} \times[0,1), \mathbb{C}^{Q}\right), j \in \mathbb{N}_{M}$, and a.e. $v \in[0,1)$ by Lemma 9. It is obvious that (94) holds if (92) holds for $j \in \mathbb{N}_{M}$ and a.e. $v \in[0,1)$. Now suppose (94) holds. For an arbitrarily fixed $x \in \mathbb{C}^{\mathrm{Q}}$, choose $d(j, v)$ as

$$
d(j, v)=x \text { for }(j, v) \in \mathbb{N}_{M} \times[0,1) .
$$

Then $d(j, v) \in L^{2}\left(\mathbb{N}_{M} \times[0,1), \mathbb{C}^{Q}\right)$, and thus $\Psi_{\mathbf{g}}^{*}(j, v) x=$ $M \Psi_{\mathbf{g}}^{*}(j, v) \Psi_{\mathbf{h}}(j, v) \Psi_{\mathbf{g}}^{*}(j, v) x$ for $j \in \mathbb{N}_{M}$ and a.e. $v \in[0,1)$ by (94). So (92) holds for $j \in \mathbb{N}_{M}$ and a.e. $v \in[0,1)$ by the arbitrariness of $x$. The proof is completed.

By Lemmas 27-29, we have the following theorem which characterizes the Gabor duals of type I (resp., type II).

Theorem 30. Given $\mathbf{g}=\left(g_{1}, g_{2}, \ldots, g_{L}\right)$ with each $g_{l} \in l^{2}(\mathbb{Z})$, let $G(\mathbf{g}, \mathbf{N}, \mathbf{M})$ be a frame for $\mathscr{M}(\mathbf{g}, \mathbf{N}, \mathbf{M})$. Then, for any Bessel sequence $G(\mathbf{h}, \mathbf{N}, \mathbf{M})$ in $l^{2}(\mathbb{Z}), G(\mathbf{h}, \mathbf{N}, \mathbf{M})$ is a Gabor dual of type I (type II) for $G(\mathbf{g}, \mathbf{N}, \mathbf{M})$ if and only if the following hold:

(i) there exists $A: \mathbb{N}_{M / q} \times[0,1) \rightarrow M_{Q, Q}\left(B: \mathbb{N}_{M / q} \times\right.$ $\left.[0,1) \rightarrow \mathscr{M}_{p, p}\right)$ such that

$\Psi_{\mathbf{h}}(j, v)=A(j, v) \Psi_{\mathbf{g}}(j, v) \quad\left(\Psi_{\mathbf{h}}(j, v)=\Psi_{\mathbf{g}}(j, v) B(j, v)\right) ;$

(ii) $\Psi_{\mathbf{g}}^{*}(j, v)=M \Psi_{\mathbf{g}}^{*}(j, v) \Psi_{\mathbf{h}}(j, v) \Psi_{\mathbf{g}}^{*}(j, v)$ for $j \in \mathbb{N}_{M / q}$ and a.e. $v \in[0,1)$. 
Theorem 31. Given $\mathbf{g}=\left(g_{1}, g_{2}, \ldots, g_{L}\right)$ with each $g_{l} \in l^{2}(\mathbb{Z})$, let $G(\mathbf{g}, \mathbf{N}, \mathbf{M})$ be a frame for $\mathscr{M}(\mathbf{g}, \mathbf{N}, \mathbf{M})$. Then, for any Bessel sequence $G(\mathbf{h}, \mathbf{N}, \mathbf{M})$ in $l^{2}(\mathbb{Z})$,

(i) $G(\mathbf{h}, \mathbf{N}, \mathbf{M})$ is a Gabor dual of type I for $G(\mathbf{g}, \mathbf{N}, \mathbf{M})$ if and only if there exists $\mathscr{A}: \mathbb{N}_{M / q} \times[0,1) \rightarrow \mathscr{M}_{\mathrm{Q}, \mathrm{Q}}$ such that

$$
\begin{aligned}
\Psi_{\mathbf{h}}(j, v)= & \frac{1}{M}\left(\Psi_{\mathbf{g}}(j, v) \Psi_{\mathbf{g}}^{*}(j, v)\right)^{\dagger} \Psi_{\mathbf{g}}(j, v) \\
& \times\left[I-M \Psi_{\mathbf{g}}^{*}(j, v) \mathscr{A}(j, v) \Psi_{\mathbf{g}}(j, v)\right] \\
& +\mathscr{A}(j, v) \Psi_{\mathbf{g}}(j, v)
\end{aligned}
$$

for $j \in \mathbb{N}_{M / q}$ and a.e. $v \in[0,1)$;

(ii) $G(\mathbf{h}, \mathbf{N}, \mathbf{M})$ is a Gabor dual of type II for $G(\mathbf{g}, \mathbf{N}, \mathbf{M})$ if

$$
\begin{aligned}
\Psi_{\mathbf{h}}(j, v)= & \frac{1}{M} \Psi_{\mathbf{g}}(j, v)\left(\Psi_{\mathbf{g}}^{*}(j, v) \Psi_{\mathbf{g}}(j, v)\right)^{\dagger} \\
& \times\left[I-M \Psi_{\mathbf{g}}^{*}(j, v) \Psi_{\mathbf{g}}(j, v) \mathscr{A}(j, v)\right] \\
& +\Psi_{\mathbf{g}}(j, v) \mathscr{A}(j, v)
\end{aligned}
$$

for $j \in \mathbb{N}_{M / q}$ and a.e. $v \in[0,1)$;

(iii) $G(\mathbf{h}, \mathbf{N}, \mathbf{M})$ is an oblique dual of $G(\mathbf{g}, \mathbf{N}, \mathbf{M})$ if one of the following conditions holds:

(a) $\Psi_{\mathbf{h}}(j, v)=(1 / M) \Psi_{\mathbf{g}}(j, v)\left(\Psi_{\mathbf{g}}^{*}(j, v) \Psi_{\mathbf{g}}(j, v)\right)^{\dagger}[I-$ $\left.M \Psi_{\mathbf{g}}^{*}(j, v) \mathscr{A}(j, v) \Psi_{\mathbf{g}}(j, v)\right]+\mathscr{A}(j, v) \Psi_{\mathbf{g}}(j, v)$ for $j \in \mathbb{N}_{M / q}$ and a.e. $v \in[0,1)$;

(b) $\Psi_{\mathbf{h}}(j, v)=(1 / M)\left(\Psi_{\mathbf{g}}(j, v) \Psi_{\mathbf{g}}^{*}(j, v)\right)^{\dagger} \Psi_{\mathbf{g}}(j, v)[I-$ $\left.M \Psi_{\mathbf{g}}^{*}(j, v) \Psi_{\mathbf{g}}(j, v) \mathscr{A}(j, v)\right]+\Psi_{\mathbf{g}}(j, v) \mathscr{A}(j, v)$ for $j \in \mathbb{N}_{M / q}$ and a.e. $v \in[0,1)$.

Proof. The items (ii) and (iii) can be proved similarly to item (i). Next we prove item (i). First we assume that (97) holds. Then

$$
\begin{aligned}
M \Psi_{\mathbf{g}}^{*} & (j, v) \Psi_{\mathbf{h}}(j, v) \Psi_{\mathbf{g}}^{*}(j, v) \\
= & \Psi_{\mathbf{g}}^{*}(j, v)\left(\Psi_{\mathbf{g}}(j, v) \Psi_{\mathbf{g}}^{*}(j, v)\right)^{\dagger} \\
& \times \Psi_{\mathbf{g}}(j, v) \Psi_{\mathbf{g}}^{*}(j, v) \\
& -M \Psi_{\mathbf{g}}^{*}(j, v)\left(\Psi_{\mathbf{g}}(j, v) \Psi_{\mathbf{g}}^{*}(j, v)\right)^{\dagger} \\
& \times \Psi_{\mathbf{g}}(j, v) \Psi_{\mathbf{g}}^{*}(j, v) \mathscr{A}(j, v) \\
& \times \Psi_{\mathbf{g}}(j, v) \Psi_{\mathbf{g}}^{*}(j, v) \\
& +M \Psi_{\mathbf{g}}^{*}(j, v) \mathscr{A}(j, v) \Psi_{\mathbf{g}}(j, v) \Psi_{\mathbf{g}}^{*}(j, v)
\end{aligned}
$$

for $j \in \mathbb{N}_{M / q}$ and a.e. $v \in[0,1)$. Also observe that $\left(\Psi_{\mathbf{g}}(j\right.$, $\left.v) \Psi_{\mathbf{g}}^{*}(j, v)\right)^{\dagger} \Psi_{\mathbf{g}}(j, v) \Psi_{\mathbf{g}}^{*}(j, v)$ is the projection from $\mathbb{C}^{\mathrm{Q}}$ onto range $\left(\Psi_{\mathbf{g}}(j, v)\right)$. It follows that $M \Psi_{\mathbf{g}}^{*}(j, v) \Psi_{\mathbf{h}}(j, v) \Psi_{\mathbf{g}}^{*}(j, v)=$ $\Psi_{\mathbf{g}}^{*}(j, v)$, and thus $G(\mathbf{h}, \mathbf{N}, \mathbf{M})$ is a Gabor dual of type I for $G(\mathbf{g}, \mathbf{N}, \mathbf{M})$ by Theorem 30 .
Now we turn to the converse implication. Suppose $G(\mathbf{h}, \mathbf{N}, \mathbf{M})$ is a Gabor dual of type I for $G(\mathbf{g}, \mathbf{N}, \mathbf{M})$. Then there exists $B(j, v): \mathbb{N}_{M / q} \times[0,1) \rightarrow \mathscr{M}_{\mathrm{Q}, \mathrm{Q}}$ such that

$$
\begin{gathered}
\Psi_{\mathbf{h}}(j, v)=B(j, v) \Psi_{\mathbf{g}}(j, v), \\
\Psi_{\mathbf{g}}^{*}(j, v)=M \Psi_{\mathbf{g}}^{*}(j, v) \Psi_{\mathbf{h}}(j, v) \Psi_{\mathbf{g}}^{*}(j, v)
\end{gathered}
$$

for $j \in \mathbb{N}_{M / q}$ and a.e. $v \in[0,1)$. It follows that

$$
\begin{aligned}
& \frac{1}{M}(\left(\Psi_{\mathbf{g}}(j, v) \Psi_{\mathbf{g}}^{*}(j, v)\right)^{\dagger} \Psi_{\mathbf{g}}(j, v) \Psi_{\mathbf{g}}^{*}(j, v) \\
&=\left(\Psi_{\mathbf{g}}(j, v) \Psi_{\mathbf{g}}^{*}(j, v)\right)^{\dagger} \Psi_{\mathbf{g}}(j, v) \\
& \quad \times \Psi_{\mathbf{g}}^{*}(j, v) \Psi_{\mathbf{h}}(j, v) \Psi_{\mathbf{g}}^{*}(j, v)
\end{aligned}
$$

for $j \in \mathbb{N}_{M / q}$ and a.e. $v \in[0,1)$, and thus

$$
\begin{aligned}
& \frac{1}{M}\left(\Psi_{\mathbf{g}}(j, v) \Psi_{\mathbf{g}}^{*}(j, v)\right)^{\dagger} \Psi_{\mathbf{g}}(j, v) \\
& =\left(\Psi_{\mathbf{g}}(j, v) \Psi_{\mathbf{g}}^{*}(j, v)\right)^{\dagger} \\
& \quad \times \Psi_{\mathbf{g}}(j, v) \Psi_{\mathbf{g}}^{*}(j, v) \Psi_{\mathbf{h}}(j, v)
\end{aligned}
$$

for $j \in \mathbb{N}_{M / q}$ and a.e. $v \in[0,1)$ due to the fact that $\mathbb{C}^{p}=\operatorname{range}\left(\Psi_{\mathbf{g}}^{*}(j, v)\right) \oplus \operatorname{ker}\left(\Psi_{\mathbf{g}}(j, v)\right)$. Put $\mathscr{A}(j, v)=B(j, v)-$ $\left(\Psi_{\mathbf{g}}(j, v) \Psi_{\mathbf{g}}^{*}(j, v)\right)^{\dagger}$. Then the right-hand side of (97) equals

$$
\begin{gathered}
\Psi_{\mathbf{h}}(j, v)+\left(\Psi_{\mathbf{g}}(j, v) \Psi_{\mathbf{g}}^{*}(j, v)\right)^{\dagger} \mathscr{P}_{\text {range }\left(\Psi_{\mathbf{g}}(j, v)\right)} \Psi_{\mathbf{g}}(j, v) \\
-\left(\Psi_{\mathbf{g}}(j, v) \Psi_{\mathbf{g}}^{*}(j, v)\right)^{\dagger} \Psi_{\mathbf{g}}(j, v)
\end{gathered}
$$

by (102), and this is exactly $\Psi_{\mathbf{h}}(j, v)$ for $j \in \mathbb{N}_{M / q}$ and a.e. $v \in[0,1)$ again by the fact that

$$
\Psi_{\mathbf{g}}(j, v) \Psi_{\mathbf{g}}^{*}(j, v)\left(\Psi_{\mathbf{g}}(j, v) \Psi_{\mathbf{g}}^{*}(j, v)\right)^{\dagger}=\mathscr{P}_{\text {range }\left(\Psi_{\mathbf{g}}(j, v)\right)} .
$$

\section{The Case of $N_{1}=N_{2}=\cdots=N_{L}$}

Theorems 30 and 31 characterize duals with Gabor structure for general $\mathbf{N}=\left(N_{1}, N_{2}, \ldots, N_{L}\right)$. This section deals with the case $N_{1}=N_{2}=\cdots=N_{L}$. We start with a remark on the case of $N_{l}, 1 \leq l \leq L$, being not all the same.

Remark 32. Not every subspace Gabor frame $G(\mathbf{g}, \mathbf{N}, \mathbf{M})$ admits an oblique Gabor dual.

We show it by revisiting Example 25. Let us make an additional assumption that $\lambda(0, v)=\lambda(1, v)=0$ and $a(0$, $v) a(1, v) \neq 0$ for $v \in[0,1]$. Then $G(\mathbf{g}, \mathbf{N}, \mathbf{M})$ is a frame but not a Riesz basis for $\mathscr{M}(\mathbf{g}, \mathbf{N}, \mathbf{M})$ by Remark 26. Suppose $G(\mathbf{h}, \mathbf{N}, \mathbf{M})$ with $\mathbf{h}=\left(h_{1}, h_{2}\right)$ is an oblique Gabor dual for $G(\mathbf{g}, \mathbf{N}, \mathbf{M})$. Then

$$
\begin{array}{r}
\Psi_{\mathbf{g}}^{*}(j, v)=2 \Psi_{\mathbf{g}}^{*}(j, v) \Psi_{\mathbf{h}}(j, v) \Psi_{\mathbf{g}}^{*}(j, v) \\
\text { for } j \in \mathbb{N}_{2}, \text { a.e. } v \in[0,1)
\end{array}
$$


by Theorem 30 . Writing out $(0,0),(3,1)$, and $(5,4)$ entries of both sides, we have

$$
\begin{gathered}
2(\overline{a(j, v)})^{2} \mathscr{Z}_{12} h_{1}(j, v)=\overline{a(j, v)}, \\
2(\overline{a(j, v)})^{2} \mathscr{Z}_{12} h_{1}(j, v) \\
+2|a(j, v)|^{2} \mathscr{Z}_{12} h_{2}(j+2, v)=\overline{a(j, v)}, \\
2(a(j, v))^{2} \mathscr{Z}_{12} h_{2}(j+2, v)=a(j, v)
\end{gathered}
$$

for $j \in \mathbb{N}_{2}$ and a.e. $v \in[0,1)$. By (107) and (108), we have $2 \overline{a(j, v)} \mathscr{Z}_{12} h_{1}(j, v)=0$ for $j \in \mathbb{N}_{2}$ and a.e. $v \in[0,1)$. This contradicts (106).

Observe that $N_{1} \neq N_{2}$ in Remark $32\left(N_{1}=6\right.$ and $\left.N_{2}=4\right)$. It is natural to ask the following question.

Does every subspace Gabor frame $G(\mathbf{g}, \mathbf{N}, \mathbf{M})$ admit no oblique Gabor dual whenever $N_{l}, 1 \leq l \leq L$, are not all the same?

The following example gives a negative answer to this question.

Example 33. Let $\mathbf{N}=(1,2)$ and $\mathbf{M}=(3,3)$. Assume that $\mathscr{J}(v)=\left(\begin{array}{l}\mathscr{I}_{1}(v) \\ \mathscr{F}_{2}(v)\end{array}\right), \mathscr{E}(v)=\left(\begin{array}{l}\mathscr{E}_{1}(v) \\ \mathscr{E}_{2}(v)\end{array}\right)$ have the form

$$
\mathscr{J}_{1}(v)=\left(\begin{array}{cc}
a_{0,0}(v) & a_{0,1}(v) \\
a_{1,0}(v) & a_{1,1}(v) \\
a_{2,0}(v) & a_{2,1}(v) \\
a_{2,1}(v) & e^{-2 \pi i v} a_{2,0}(v) \\
e^{2 \pi i v} a_{0,1}(v) & a_{0,0}(v) \\
e^{2 \pi i v} a_{1,1}(v) & a_{1,0}(v) \\
c_{0,0}(v) & c_{0,1}(v) \\
c_{1,0}(v) & c_{1,1}(v) \\
c_{2,0}(v) & c_{2,1}(v) \\
c_{2,1}(v) & e^{-2 \pi i v} c_{2,0}(v) \\
e^{2 \pi i v} c_{0,1}(v) & c_{0,0}(v) \\
e^{2 \pi i v} c_{1,1}(v) & c_{1,0}(v) \\
b_{0,0}(v) & b_{0,1}(v) \\
b_{1,0}(v) & b_{1,1}(v) \\
b_{2,0}(v) & b_{2,1}(v)
\end{array}\right),
$$

for $v \in[0,1)$ with all entries of $\mathscr{J}(v)$ and $\mathscr{E}(v)$ in $L^{\infty}([0,1))$, that $\left(\mathscr{J}_{1}(v)\right)^{*} \mathscr{E}_{1}(v)$ has the form $\left(\begin{array}{cc}A(v) & 0 \\ 0 & A(v)\end{array}\right)$ and $(\mathscr{J}(v))^{*} \mathscr{J}(v)$ has the form $\left(\begin{array}{cc}B(v) & 0 \\ 0 & B(v)\end{array}\right)$ for $v \in[0,1)$, and that

$$
\left(\mathscr{J}_{2}(v)\right)^{*} \mathscr{E}_{2}(v)=\left(\begin{array}{cc}
\frac{1}{3}-A(v) & 0 \\
0 & \frac{1}{3}-A(v)
\end{array}\right)
$$

for $v \in[0,1)$ satisfying $B(v) \neq 0$. Define $\mathbf{g}=\left(g_{1}, g_{2}\right)$ and $\mathbf{h}=$ $\left(h_{1}, h_{2}\right)$ by

$$
\Psi_{\mathbf{g}}(0, v)=\mathscr{J}(v), \quad \Psi_{\mathbf{h}}(0, v)=\mathscr{E}(v) \quad \text { for } v \in[0,1) .
$$

Then $\mathbf{g}$ and $\mathbf{h}$ are well defined by the quasi-periodicity of the Zak transform $\mathscr{Z}_{6}$ and $6 \mathbb{Z}$ congruence between $\{0\}-2 \mathbb{N}_{3}+$ $3 \mathbb{N}_{2}$ and $\mathbb{N}_{6}$, and $G(\mathbf{g}, \mathbf{N}, \mathbf{M})$ and $G(\mathbf{h}, \mathbf{N}, \mathbf{M})$ are both Bessel sequences by Remark 16. A simple computation shows that

$$
\Psi_{\mathbf{g}}^{*}(0, v) \Psi_{\mathbf{g}}(0, v)=3 \Psi_{\mathbf{g}}^{*}(0, v) \Psi_{\mathbf{h}}(0, v) \Psi_{\mathbf{g}}^{*}(0, v) \Psi_{\mathbf{g}}(0, v)
$$

for $v \in[0,1)$. This implies that $\Psi_{\mathbf{g}}^{*}(0, v)=3 \Psi_{\mathbf{g}}^{*}(0, v) \Psi_{\mathbf{h}}(0$, v) $\Psi_{\mathbf{g}}^{*}(0, v)$ for $v \in[0,1)$ due to the fact that $\mathbb{C}^{9}=$ $\operatorname{range}\left(\Psi_{\mathbf{g}}(0, v)\right) \oplus \operatorname{ker}\left(\Psi_{\mathbf{g}}^{*}(0, v)\right)$. So $G(\mathbf{h}, \mathbf{N}, \mathbf{M})$ is an oblique Gabor dual for $G(\mathbf{h}, \mathbf{N}, \mathbf{M})$ by Lemma 29.

Remark 32 and Example 33 show that the Gabor dual theory for subspace Gabor frames is complicated when $N_{l}$, $1 \leq l \leq L$, are not all the same. There are still many unresolved problems in this direction. Next we work under the following assumption.

Assumption 34. $L$ is a positive integer, $\mathbf{M}=(M, M, \ldots, M)$, and $\mathbf{N}=\left(N_{1}, N_{2}, \ldots, N_{L}\right)$ with $N_{1}=N_{2}=\cdots=N_{L}=N$.

The following theorem characterizes the uniqueness of such duals.

Theorem 35. Given $\mathbf{g}=\left(g_{1}, g_{2}, \ldots, g_{L}\right)$ with each $g_{l} \in l^{2}(\mathbb{Z})$, let $G(\mathbf{g}, \mathbf{N}, \mathbf{M})$ be a frame for $\mathscr{M}(\mathbf{g}, \mathbf{N}, \mathbf{M})$. Then the following are equivalent:

(i) $G(\mathbf{g}, \mathbf{N}, \mathbf{M})$ has a unique Gabor dual of type I (type II);

(ii) $\operatorname{rank}\left(\Psi_{\mathbf{g}}(j, v)\right) \in\{0, L q\}\left(\operatorname{rank}\left(\Psi_{\mathbf{g}}(j, v)\right) \in\{0, p\}\right)$ for a.e. $(j, v) \in \mathbb{N}_{M / q} \times[0,1)$;

(iii) $\operatorname{rank}\left(\Psi_{\mathbf{g}}(j, v)\right) \in\{0, L q\}\left(\operatorname{rank}\left(\Psi_{\mathbf{g}}(j, v)\right) \in\{0, p\}\right)$ for a.e. $(j, v) \in \mathbb{Z} \times \mathbb{R}$.

Proof. We first deal with the Gabor dual of type I. By Lemma 12, (ii) and (iii) are equivalent. By Theorem 30 and Lemma 27, we only need to prove that (ii) holds if and only if a function $A: \mathbb{N}_{M} \times[0,1) \rightarrow \mathscr{M}_{L q, L q}$ solves

$$
A(j, v) \Psi_{\mathbf{g}}(j, v)=0 \quad \text { for } j \in \mathbb{N}_{M} \text {, a.e. } v \in[0,1)
$$

whenever it solves

$$
\begin{array}{r}
\Psi_{\mathbf{g}}^{*}(j, v) A(j, v) \Psi_{\mathbf{g}}(j, v) \Psi_{\mathbf{g}}^{*}(j, v)=0 \\
\text { for } j \in \mathbb{N}_{M}, \text { a.e. } v \in[0,1)
\end{array}
$$


Suppose (ii) holds and $A: \mathbb{N}_{M} \times[0,1) \rightarrow \mathscr{M}_{L q, L q}$ solves (114). If $(j, v)$ is such that $\operatorname{rank}\left(\Psi_{\mathbf{g}}(j, v)\right)=0$, then $\Psi_{\mathbf{g}}(j, v)=0$, and thus $A(j, v) \Psi_{\mathbf{g}}(j, v)=0$. If $(j, v)$ is such that $\operatorname{rank}\left(\Psi_{\mathbf{g}}(j, v)\right)=$ $L q$, then (114) implies that

$$
A(j, v) \Psi_{\mathbf{g}}(j, v) \Psi_{\mathbf{g}}^{*}(j, v)=0,
$$

which leads to $A(j, v)=0$ and thus $A(j, v) \Psi_{\mathbf{g}}(j, v)=0$. Conversely, suppose (ii) does not hold. Then there exist some $j_{0} \in \mathbb{N}_{M}$ and $\mathbf{e}_{k}, \mathbf{e}_{r}$ such that

$$
\mathscr{P}\left(j_{0}, v\right) \mathbf{e}_{k} \neq 0, \quad \mathbf{e}_{r}^{*} \Psi_{\mathbf{g}}\left(j_{0}, v\right) \neq 0
$$

on some $E \subset[0,1)$ with $|E|>0$, where $\mathscr{P}\left(j_{0}, v\right)$ is the orthogonal projection of $\mathbb{C}^{L q}$ onto $\operatorname{ker}\left(\Psi_{\mathrm{g}}^{*}\left(j_{0}, v\right)\right)$. Define

$$
A\left(j_{0}, v\right)= \begin{cases}\mathscr{P}\left(j_{0}, v\right) \mathbf{e}_{k} \mathbf{e}_{r}^{*}, & \text { if } j=j_{0}, v \in E \\ 0, & \text { otherwise }\end{cases}
$$

for $(j, v) \in \mathbb{N}_{M} \times[0,1)$. Then (114) holds, but (113) fails to hold.

Next we turn to the Gabor duals of type II. Similarly to the above arguments, by Theorem 30 and Lemmas 27 and 28, we only need to prove that (ii) holds if and only if a function $B: \mathbb{N}_{M} \times[0,1) \rightarrow \mathscr{M}_{p, p}$ solves

$$
\Psi_{\mathbf{g}}(j, v) B(j, v)=0 \quad \text { for } j \in \mathbb{N}_{M} \text {, a.e. } v \in[0,1)
$$

whenever it solves

$$
\begin{array}{r}
\Psi_{\mathbf{g}}^{*}(j, v) \Psi_{\mathbf{g}}(j, v) B(j, v) \Psi_{\mathbf{g}}^{*}(j, v)=0 \\
\text { for } j \in \mathbb{N}_{M}, \text { a.e. } v \in[0,1) .
\end{array}
$$

The necessity can be proved similarly to the case of type I. Now we suppose (ii) does not hold. Then there exist some $j_{0} \in \mathbb{N}_{M}$ and $\mathbf{e}_{k}, \mathbf{e}_{r}$ such that

$$
\mathscr{P}\left(j_{0}, v\right) \mathbf{e}_{k} \neq 0, \quad \Psi_{\mathbf{g}}\left(j_{0}, v\right) \mathbf{e}_{r} \neq 0
$$

on some $E \subset[0,1)$ with $|E|>0$, where $\mathscr{P}\left(j_{0}, v\right)$ is the orthogonal projection of $\mathbb{C}^{p}$ onto $\operatorname{ker}\left(\Psi_{\mathbf{g}}\left(j_{0}, v\right)\right)$. Define $B\left(j_{0}, v\right)$ by

$$
B^{*}\left(j_{0}, v\right)= \begin{cases}\mathscr{P}\left(j_{0}, v\right) \mathbf{e}_{k} \mathbf{e}_{r}^{*}, & \text { if } j=j_{0}, v \in E, \\ 0, & \text { otherwise }\end{cases}
$$

for $(j, v) \in \mathbb{N}_{M} \times[0,1)$. Then (119) holds, but (118) fails to hold. The proof is completed.

It is well known that every scalar $k \times n$ matrix $M$ with rank $r \geq 1$ has a decomposition

$$
M=U\left(\begin{array}{ll}
D & 0 \\
0 & 0
\end{array}\right) V^{*}
$$

where $U$ and $V$ are, respectively, $k \times k$ and $n \times n$ unitary matrices, $\left(\begin{array}{ll}D & 0 \\ 0 & 0\end{array}\right)$ is a $k \times n$ block matrix in which $D$ is an $r \times r$ diagonal matrix with positive entries in the diagonal (see [1, Theorem 1.5.4]). Observe that $\operatorname{rank}(M)$ may change in variables if $M$ is a matrix-valued function. Next we restrict ourselves to Gabor systems $G(\mathbf{g}, \mathbf{N}, \mathbf{M})$ such that

$$
\begin{array}{r}
\Psi_{\mathbf{g}}(j, v)=U(j, v)\left(\begin{array}{cc}
D(j, v) & 0 \\
0 & 0
\end{array}\right) V^{*}(j, v) \\
\text { for } j \in \mathbb{N}_{M / q}, \text { a.e. } v \in[0,1),
\end{array}
$$

where $U(j, v)$ and $V(j, v)$ are, respectively, $L q \times L q$ and $p \times p$ unitary matrices for $j \in \mathbb{N}_{M / q}$ and a.e. $v \in[0,1)$, and $\left(\begin{array}{cc}\mathrm{D}(j, v) & 0 \\ 0 & 0\end{array}\right)$ is an $L q \times p$ block matrix in which $D(j, v)$ is of the form

$$
D(j, v)=\operatorname{diag}\left(\lambda_{1}(j, v), \lambda_{2}(j, v), \ldots, \lambda_{m}(j, v)\right)
$$

with $\lambda_{i}(j, v) \geq 0$ for $j \in \mathbb{N}_{M / q}$ and a.e. $v \in[0,1)$ and $\bigcup_{j \in \mathbb{N}_{M / q}}\left\{v \in[0,1): \lambda_{i}(j, v)>0\right\}$ being of positive measure for each $1 \leq i \leq m$.

As an immediate consequence of Theorems 19, 24, and 35, we have the following theorem.

Theorem 36. Let $\mathbf{0} \neq \mathbf{g}=\left(g_{1}, g_{2}, \ldots, g_{L}\right)$ be defined as in (123). Then

(i) $G(\mathbf{g}, \mathbf{N}, \mathbf{M})$ is a frame for $\mathscr{M}(\mathbf{g}, \mathbf{N}, \mathbf{M})$ with frame bounds $A, B$ if and only if

$$
\begin{aligned}
\sqrt{\frac{A}{M}} & \leq \lambda_{i}(j, v) \\
& \leq \sqrt{\frac{B}{M}} \text { on }\left\{(j, v) \in \mathbb{N}_{M / q} \times[0,1): \lambda_{i}(j, v)>0\right\}
\end{aligned}
$$

for $1 \leq i \leq m$;

(ii) $G(\mathbf{g}, \mathbf{N}, \mathbf{M})$ is a Riesz basis (an orthonormal basis) for $\mathscr{M}(\mathbf{g}, \mathbf{N}, \mathbf{M})$ with Riesz bounds $A, B$ if and only if

$m=L q, \quad \sqrt{\frac{A}{M}} \leq \lambda_{i}(j, v) \leq \sqrt{\frac{B}{M}} \quad\left(\lambda_{i}(j, v)=\sqrt{\frac{1}{M}}\right)$

for $1 \leq i \leq m, j \in \mathbb{N}_{M / q}$, and a.e. $v \in[0,1)$;

(iii) $G(\mathbf{g}, \mathbf{N}, \mathbf{M})$ is a frame for $\mathscr{M}(\mathbf{g}, \mathbf{N}, \mathbf{M})$ with frame bounds $A$ and $B$ and simultaneously admits a unique Gabor dual of type I (type II) if and only if $m=L q$ $(m=p)$, and for each $j \in \mathbb{N}_{M / q}$ and a.e. $v \in[0,1)$, either $\lambda_{i}(j, v)=0$ for each $1 \leq i \leq m$ or $\sqrt{A / M} \leq$ $\lambda_{i}(j, v) \leq \sqrt{B / M}$ for each $1 \leq i \leq m$.

Theorem 37. Let $\mathbf{g}$ be defined as in (123) and let $G(\mathbf{g}, \mathbf{N}, \mathbf{M})$ be a frame for $\mathscr{M}(\mathbf{g}, \mathbf{N}, \mathbf{M})$. Then, for $\mathbf{h}=\left(h_{l}, h_{2}, \ldots, h_{L}\right)$ with each $h_{l} \in l^{2}(\mathbb{Z})$, we have

(i) $G(\mathbf{h}, \mathbf{N}, \mathbf{M})$ is a Gabor dual of type I for $G(\mathbf{g}, \mathbf{N}, \mathbf{M})$ if and only if $\Psi_{\mathbf{h}}$ has the form

$$
\begin{array}{r}
\Psi_{\mathbf{h}}(j, v)=\frac{1}{M} U(j, v) C(j, v)\left(\begin{array}{cc}
D(j, v) & 0 \\
0 & 0
\end{array}\right) V^{*}(j, v) \\
\text { for } j \in \mathbb{N}_{M / q}, \text { a.e. } v \in[0,1),
\end{array}
$$


where $C: \mathbb{N}_{M / q} \times[0,1) \rightarrow M_{L q, L q}$ satisfies $C(j, v)=$ $\left(C_{1}(j, v), 0\right)$ with $C_{1}(j, v) \in \mathscr{M}_{L q, m}$, the entries of the ith column of $C_{1}(j, v)$ are essentially bounded on $\left\{(j, v) \in \mathbb{N}_{M / q} \times[0,1): \lambda_{i}(j, v)>0\right\}$ for $1 \leq i \leq m$, and

$$
(C(j, v))_{i, n}= \begin{cases}0, & i \neq n, \lambda_{i}(j, v) \lambda_{n}(j, v) \neq 0 \\ \left(\lambda_{i}(j, v)\right)^{-2}, & i=n, \lambda_{i}(j, v) \neq 0\end{cases}
$$

for $1 \leq i, n \leq m$;

(ii) $G(\mathbf{h}, \mathbf{N}, \mathbf{M})$ is a Gabor dual of type II for $G(\mathbf{g}, \mathbf{N}, \mathbf{M})$ if and only if $\Psi_{\mathbf{h}}$ has the form

$$
\begin{array}{r}
\Psi_{\mathbf{h}}(j, v)=\frac{1}{M} U(j, v)\left(\begin{array}{cc}
D(j, v) & 0 \\
0 & 0
\end{array}\right) C(j, v) V^{*}(j, v) \\
\text { for } j \in \mathbb{N}_{M / q}, \text { a.e. } v \in[0,1),
\end{array}
$$

where $C: \mathbb{N}_{M / q} \times[0,1) \rightarrow M_{p, p}$ satisfies $C(j, v)=\left(\begin{array}{c}C_{1}(j, v) \\ 0\end{array}\right)$ with $C_{1}(j, v) \in \mathscr{M}_{m, p}$, the entries of the ith row of $C_{1}(j, v)$ are essentially bounded on $\left\{(j, v) \in \mathbb{N}_{M / q} \times[0,1): \lambda_{i}(j, v)>0\right\}$ for $1 \leq i \leq m$, and

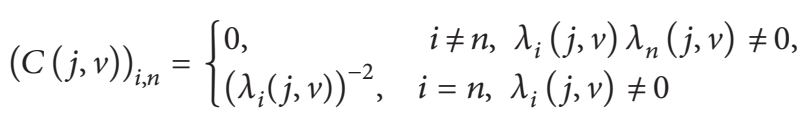

for $1 \leq i, n \leq m$.

Proof. We only prove item (i), and (ii) can be proved similarly. By Theorem 30, $G(\mathbf{h}, \mathbf{N}, \mathbf{M})$ is a Gabor dual of type I for $G(\mathbf{g}, \mathbf{N}, \mathbf{M})$ if and only if there exists a function $A: \mathbb{N}_{M / q} \times$ $[0,1) \rightarrow \mathscr{M}_{L q, L q}$ such that

$$
\begin{gathered}
\Psi_{\mathbf{h}}(j, v)=A(j, v) \Psi_{\mathbf{g}}(j, v), \\
\Psi_{\mathbf{g}}^{*}(j, v)=M \Psi_{\mathbf{g}}^{*}(j, v) A(j, v) \Psi_{\mathbf{g}}(j, v) \Psi_{\mathbf{g}}^{*}(j, v)
\end{gathered}
$$

for $j \in \mathbb{N}_{M / q}$ and a.e. $[0,1)$, and for each $j \in \mathbb{N}_{M / q}$ the entries of $\Psi_{\mathbf{h}}(j, v)$ belong to $L^{\infty}([0,1))$. Write

$$
\begin{aligned}
C(j, v) & =M U^{*}(j, v) A(j, v) U(j, v) \\
& =\left(C_{1}(j, v), C_{2}(j, v)\right),
\end{aligned}
$$

where $C_{1}(j, v) \in \mathscr{M}_{L q, m}$. A simple computation shows that (132) is equivalent to

$$
\begin{gathered}
D(j, v)=D(j, v) C_{11}(j, v) D^{2}(j, v) \\
\text { for } j \in \mathbb{N}_{M / q}, \text { a.e. } v \in[0,1),
\end{gathered}
$$

where $C_{1}(j, v)=\left(\begin{array}{l}C_{11}(j, v) \\ C_{21}(j, v)\end{array}\right), C_{11}(j, v) \in \mathscr{M}_{m, m}$. It is obvious that (134) is equivalent to (128), which implies that (132) is equivalent to (128). By (133), (132) can be rewritten as

$$
\Psi_{\mathbf{h}}(j, v)=\frac{1}{M} U(j, v)\left(C_{1}(j, v) D(j, v), 0\right) V^{*}(j, v) .
$$

Since $C_{2}(j, v)$ does not appear in (135), we assume that $C_{2}(j, v)=0$ without loss of generality. By the unitary properties of $U(j, v)$ and $V(j, v)$, for each $j \in \mathbb{N}_{M / q}$, the entries of $\Psi_{\mathbf{h}}(j, v)$ belong to $L^{\infty}([0,1))$ if and only if the entries of $C_{1}(j, v) D(j, v)$ belong to $L^{\infty}([0,1))$; that is, the entries of the $i$ th column of $C_{1}(j, v)$ are essentially bounded on $\{(j, v) \in$ $\left.\mathbb{N}_{M / q} \times[0,1): \lambda_{i}(j, v)>0\right\}$ for $1 \leq i \leq m$. The proof is completed.

Next we discuss a special case of Theorem 36 . Let $\mathbf{N}=1$, $\mathbf{M}=2, \overrightarrow{\mathbf{1}}=(1,1)$, and $\overrightarrow{\mathbf{2}}=(2,2)$ and write $V_{1}(0, v)=\widetilde{V}(0, v)=$ 1 and

$$
\begin{gathered}
U_{1}(0, v)=\frac{1}{\sqrt{1+\cos ^{2} 2 \pi v}}\left(\begin{array}{cc}
-1 & \cos 2 \pi v \\
\cos 2 \pi v & 1
\end{array}\right), \\
D_{1}(0, v)=\left(\begin{array}{c}
\sqrt{1+\cos ^{2} 2 \pi v} \\
0
\end{array}\right)
\end{gathered}
$$

$\widetilde{U}(0, v)$

$$
\begin{gathered}
=\frac{1}{\sqrt{3}}\left(\begin{array}{cccc}
-1 & i & -i \cos 2 \pi v & -i \sin 2 \pi v \\
\cos 2 \pi v & i \sin 2 \pi v & -i & i \\
-1 & -i & -i \sin 2 \pi v & i \cos 2 \pi v \\
-i \sin 2 \pi v & -\cos 2 \pi v & -1 & -1
\end{array}\right) \\
\widetilde{D}(0, v)=\left(\begin{array}{c}
\sqrt{3} \\
0 \\
0 \\
0
\end{array}\right)
\end{gathered}
$$

for $v \in[0,1)$. Define $\mathbf{g}$ and $\widetilde{\mathbf{g}}=\left(\widetilde{\mathfrak{g}}_{1}, \widetilde{g}_{2}\right)$ as

$$
\begin{gathered}
\Psi_{\mathbf{g}}(0, v)=U_{1}(0, v) D_{1}(0, v) V_{1}^{*}(0, v), \\
\Psi_{\widetilde{\mathbf{g}}}(0, v)=\widetilde{U}(0, v) \widetilde{D}(0, v) \widetilde{V}^{*}(0, v)
\end{gathered}
$$

by letting $U=U_{1}, D=D_{1}$, and $V=V_{1}$ and $U=\widetilde{U}, D=\widetilde{D}$, and $V=\widetilde{V}$ in (123), respectively. Then

$$
\begin{gathered}
\Psi_{\mathbf{g}}(0, v)=\left(\begin{array}{c}
-1 \\
\cos 2 \pi v
\end{array}\right), \\
\Psi_{\widetilde{\mathbf{g}}}(0, v)=\left(\begin{array}{c}
-1 \\
\cos 2 \pi v \\
-1 \\
-i \sin 2 \pi v
\end{array}\right) \text { for } v \in[0,1) .
\end{gathered}
$$

This implies that $\mathbf{g}=\tilde{g}_{1}=-\chi_{\{0\}}+(1 / 2) \chi_{\{-3,1\}}, \tilde{g}_{2}=-\chi_{\{0\}}-$ $(1 / 2) \chi_{\{1\}}+(1 / 2) \chi_{\{-3\}}$ and that

$$
\mathscr{M}(\mathbf{g}, 1,2)=\mathscr{M}(\widetilde{\mathbf{g}}, \overrightarrow{\mathbf{1}}, \overrightarrow{\mathbf{2}})=l^{2}(\mathbb{Z})
$$

by [5, Theorem 3.1]. So $G(\mathbf{g}, 1,2)$ and $G(\widetilde{\mathbf{g}}, \overrightarrow{\mathbf{1}}, \overrightarrow{2})$ are both frames for $l^{2}(\mathbb{Z})$, but neither is a Riesz basis by Theorem 36 . 
Define $\mathbf{h} \in l^{2}(\mathbb{Z})$ and $\tilde{\mathbf{h}}=\left(\tilde{h}_{1}, \tilde{h}_{2}\right)$ with $\tilde{h}_{1}, \tilde{h}_{2} \in l^{2}(\mathbb{Z})$ by $\Psi_{\mathbf{h}}(0, v)=(1 / 2) \Psi_{\mathbf{g}}(0, v)\left(\Psi_{\mathbf{g}}^{*}(0, v) \Psi_{\mathbf{g}}(0, v)\right)^{\dagger}$ and $\Psi_{\widetilde{\mathbf{h}}}(0, v)=$ $(1 / 2) \Psi_{\widetilde{\mathbf{g}}}(0, v)\left(\Psi_{\widetilde{\mathbf{g}}}^{*}(0, v) \Psi_{\widetilde{\mathbf{g}}}(0, v)\right)^{\dagger}$ for $v \in[0,1)$. Then $G(\mathbf{h}, 1,2)$ and $G(\tilde{\mathbf{h}}, \overrightarrow{\mathbf{1}}, \overrightarrow{\mathbf{2}})$ are, respectively, the canonical duals for $G(\mathbf{g}, 1,2)$ and $G(\widetilde{\mathbf{g}}, \overrightarrow{\mathbf{1}}, \overrightarrow{\mathbf{2}})$ by $[5$, Remark 5.5], and

$$
\begin{gathered}
\Psi_{\mathbf{h}}(0, v)=\frac{1}{2\left(1+\cos ^{2} 2 \pi v\right)}\left(\begin{array}{c}
-1 \\
\cos 2 \pi v
\end{array}\right), \\
\Psi_{\widetilde{\mathbf{h}}}(0, v)=\frac{1}{6}\left(\begin{array}{c}
-1 \\
\cos 2 \pi v \\
-1 \\
-i \sin 2 \pi v
\end{array}\right)
\end{gathered}
$$

for $v \in[0,1)$. It follows that

$$
\widetilde{\mathbf{h}}=\left(-\frac{1}{6} \chi_{\{0\}}+\frac{1}{12} \chi_{\{-3,1\}},-\frac{1}{6} \chi_{\{0\}}-\frac{1}{12} \chi_{\{1\}}+\frac{1}{12} \chi_{\{-3\}}\right),
$$

and $\mathbf{h}$ is infinitely supported. It is interesting that $\widetilde{\mathbf{g}}$ and $\widetilde{\mathbf{h}}$ are both finitely supported, but $\mathbf{h}$ is not although $\mathbf{g}$ is the first component of $\widetilde{\mathbf{g}}$. Therefore, there exist significant differences in Gabor duals between multi-window Gabor frames and single-window ones. Now we conclude this paper by summarizing the above arguments as the following example.

Example 38. Let $\overrightarrow{\mathbf{1}}=(1,1)$ and $\overrightarrow{\mathbf{2}}=(2,2)$,

$$
\begin{gathered}
\mathbf{g}=\widetilde{g}_{1}=-\chi_{\{0\}}+\frac{1}{2} \chi_{\{-3,1\}}, \\
\widetilde{g}_{2}=-\chi_{\{0\}}-\frac{1}{2} \chi_{\{1\}}+\frac{1}{2} \chi_{\{-3\}} .
\end{gathered}
$$

$\mathbf{h}$ and $\widetilde{\mathbf{h}}$, as in (140) and (142) and write $\widetilde{\mathbf{g}}=\left(\widetilde{g}_{1}, \widetilde{g}_{2}\right)$. Then

(i) $G(\mathbf{g}, 1,2)$ and $G(\widetilde{\mathbf{g}}, \overrightarrow{\mathbf{1}}, \overrightarrow{2})$ are both frames for $l^{2}(\mathbb{Z})$, but neither is a Riesz basis for $l^{2}(\mathbb{Z})$;

(ii) $G(\mathbf{h}, 1,2)$ and $G(\widetilde{\mathbf{h}}, \overrightarrow{\mathbf{1}}, \overrightarrow{\mathbf{2}})$ are, respectively, the canonical duals for $G(\mathbf{g}, 1,2)$ and $G(\widetilde{\mathbf{g}}, \overrightarrow{\mathbf{1}}, \overrightarrow{\mathbf{2}})$;

(iii) $\widetilde{\mathbf{h}}$ is finitely supported, and $\mathbf{h}$ is not.

\section{Acknowledgments}

This work is supported by the National Natural Science Foundation of China (Grant no. 11271037), Beijing Natural Science Foundation (Grant no. 1122008), and the Scientific Research Common Program of Beijing Municipal Commission of Education (Grant no. KM201110005030).

\section{References}

[1] O. Christensen, An Introduction to Frames and Riesz Bases, Applied and Numerical Harmonic Analysis, Birkhäuser, Boston, Mass, USA, 2003.

[2] H. G. Feichtinger and T. Strohmer, Gabor Analysis and Algorithms: Theory and applications, Applied and Numerical Harmonic Analysis, Birkhäuser, Boston, Mass, USA, 1998.
[3] H. G. Feichtinger and T. Strohmer, Advances in Gabor analysis, Applied and Numerical Harmonic Analysis, Birkhäuser Boston Inc., Boston, Mass, USA, 2003.

[4] R. M. Young, An Introduction to Nonharmonic Fourier Series, vol. 93 of Pure and Applied Mathematics, Academic Press, New York, NY, USA, 1980.

[5] Y.-Z. Li and Q.-F. Lian, "Multi-window Gabor frames and oblique Gabor duals on discrete periodic sets," Science China Mathematics, vol. 54, no. 5, pp. 987-1010, 2011.

[6] Q.-F. Lian and Y.-Z. Li, "The duals of Gabor frames on discrete periodic sets," Journal of Mathematical Physics, vol. 50, no. 1, article 013534, p. 22, 2009.

[7] M. A. Akinlar and J.-P. Gabardo, "Oblique duals associated with rational subspace Gabor frames," Journal of Integral Equations and Applications, vol. 20, no. 3, pp. 283-309, 2008.

[8] J.-P. Gabardo and D. Han, "Balian-Low phenomenon for subspace Gabor frames," Journal of Mathematical Physics, vol. 45, no. 8, pp. 3362-3378, 2004.

[9] K. Gröchenig, Foundations of Time-Frequency Analysis, Applied and Numerical Harmonic Analysis, Birkhäuser, Boston, Mass, USA, 2001.

[10] C. Heil, "History and evolution of the density theorem for Gabor frames," The Journal of Fourier Analysis and Applications, vol. 13, no. 2, pp. 113-166, 2007.

[11] M. Zibulski and Y. Y. Zeevi, "Analysis of multiwindow Gabortype schemes by frame methods," Applied and Computational Harmonic Analysis, vol. 4, no. 2, pp. 188-221, 1997.

[12] H. G. Feichtinger and D. M. Onchiş, "Constructive reconstruction from irregular sampling in multi-window spline-type spaces," in Progress in Analysis and Its Applications, pp. 257-265, World Scientific Publishing, Hackensack, NJ, USA, 2010.

[13] H. G. Feichtinger and D. M. Onchis, "Constructive realization of dual systems for generators of multi-window spline-type spaces," Journal of Computational and Applied Mathematics, vol. 234, no. 12, pp. 3467-3479, 2010.

[14] F. Jaillet and B. Torrésani, "Time-frequency jigsaw puzzle: adaptive multiwindow and multilayered Gabor expansions," International Journal of Wavelets, Multiresolution and Information Processing, vol. 5, no. 2, pp. 293-315, 2007.

[15] S. Li, "Discrete multi-Gabor expansions," IEEE Transactions on Information Theory, vol. 45, no. 6, pp. 1954-1967, 1999.

[16] Y. Y. Zeevi, "Multiwindow Gabor-type representations and signal representation by partial information," in Twentieth Century Harmonic Analysis-A Celebration, vol. 33 of Nato Science Series II, pp. 173-199, Kluwer Academic Publishers, Dordrecht, The Netherlands, 2001.

[17] Y. Y. Zeevi, M. Zibulski, and M. Porat, "Multi-window Gabor schemes in signal and image representations," in Gabor Analysis and Algorithms, Applied and Numerical Harmonic Analysis, pp. 381-407, Birkhäuser Boston, Boston, Mass, USA, 1998.

[18] P. G. Casazza and O. Christensen, "Weyl-Heisenberg frames for subspaces of $L^{2}(\mathbb{R})$," Proceedings of the American Mathematical Society, vol. 129, no. 1, pp. 145-154, 2001.

[19] J.-P. Gabardo and D. Han, "Subspace Weyl-Heisenberg frames," The Journal of Fourier Analysis and Applications, vol. 7, no. 4, pp. 419-433, 2001.

[20] J.-P. Gabardo and D. Han, "The uniqueness of the dual of Weyl-Heisenberg subspace frames," Applied and Computational Harmonic Analysis, vol. 17, no. 2, pp. 226-240, 2004.

[21] J.-P. Gabardo and Y.-Z. Li, "Density results for Gabor systems associated with periodic subsets of the real line," Journal of Approximation Theory, vol. 157, no. 2, pp. 172-192, 2009. 
[22] Q.-F. Lian and Y.-Z. Li, "Gabor frame sets for subspaces," Advances in Computational Mathematics, vol. 34, no. 4, pp. 391411, 2011.

[23] Y. Zhang and Y. -Z. Li, "Rational time-frequency multi-window subspace Gabor frames and their Gabor duals," Science China Mathematics, 2013.

[24] A. J. E. M. Janssen, "From continuous to discrete WeylHeisenberg frames through sampling," The Journal of Fourier Analysis and Applications, vol. 3, no. 5, pp. 583-596, 1997.

[25] R. S. Orr, "Derivation of the finite discrete Gabor transform by periodization and sampling," Signal Processing, vol. 34, no. 1, pp. 85-97, 1993.

[26] P. L. Søndergaard, "Gabor frames by sampling and periodization," Advances in Computational Mathematics, vol. 27, no. 4, pp. 355-373, 2007.

[27] C. Heil, “A discrete Zak transform,” Tech. Rep. MTR-89W00128, 1989.

[28] L. Auslander, I. C. Gertner, and R. Tolimieri, "The discrete Zak transform application to time-frequency analysis and synthesis of nonstationary signals," IEEE Transactions on Signal Processing, vol. 39, no. 4, pp. 825-835, 1991.

[29] H. Bölcskei and F. Hlawatsch, "Discrete zak transforms, polyphase transforms, and applications," IEEE Transactions on Signal Processing, vol. 45, no. 4, pp. 851-866, 1997.

[30] Z. Cvetković and M. Vetterli, "Tight Weyl-Heisenberg frames in $l^{2}(\mathbb{Z})$," IEEE Transactions on Signal Processing, vol. 46, no. 5, pp. 1256-1259, 1998.

[31] B. Hirosaki, "An orthogonally multiplexed QAM system using discrete Fourier transform," IEEE Transactions on Communications Systems, vol. 29, no. 7, pp. 982-989, 1981.

[32] Q.-F. Lian and Y.-Z. Li, “Gabor families in $l^{2}\left(\mathbb{Z}^{d}\right)$," Kyoto Journal of Mathematics, vol. 52, no. 1, pp. 179-204, 2012.

[33] J. M. Morris and Y. Lu, "Discrete Gabor expansion of discretetime signals in $l^{2}(\mathbb{Z})$ via frame theory," Signal Processing, vol. 40, no. 2-3, pp. 155-181, 1994.

[34] J. Wexler and S. Raz, "Discrete Gabor expansions," Signal Processing, vol. 21, no. 3, pp. 207-220, 1990.

[35] Y.-Z. Li and Q.-F. Lian, "Gabor systems on discrete periodic sets," Science in China A, vol. 52, no. 8, pp. 1639-1660, 2009.

[36] I. Daubechies, "The wavelet transform, time-frequency localization and signal analysis," IEEE Transactions on Information Theory, vol. 36, no. 5, pp. 961-1005, 1990. 


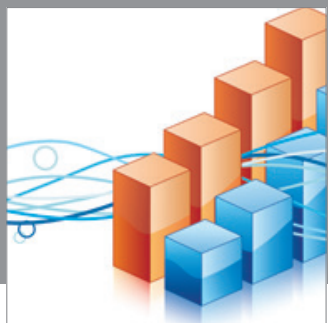

Advances in

Operations Research

mansans

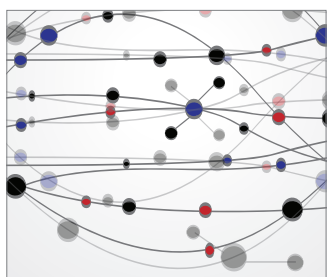

The Scientific World Journal
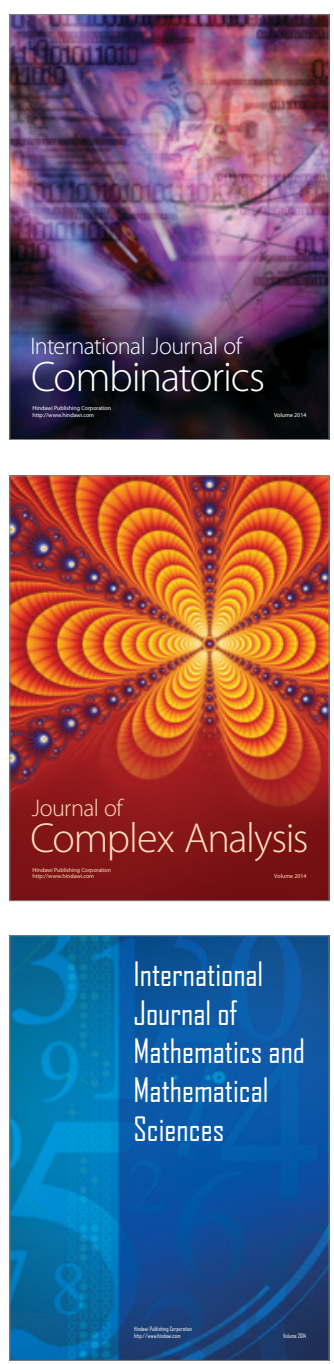
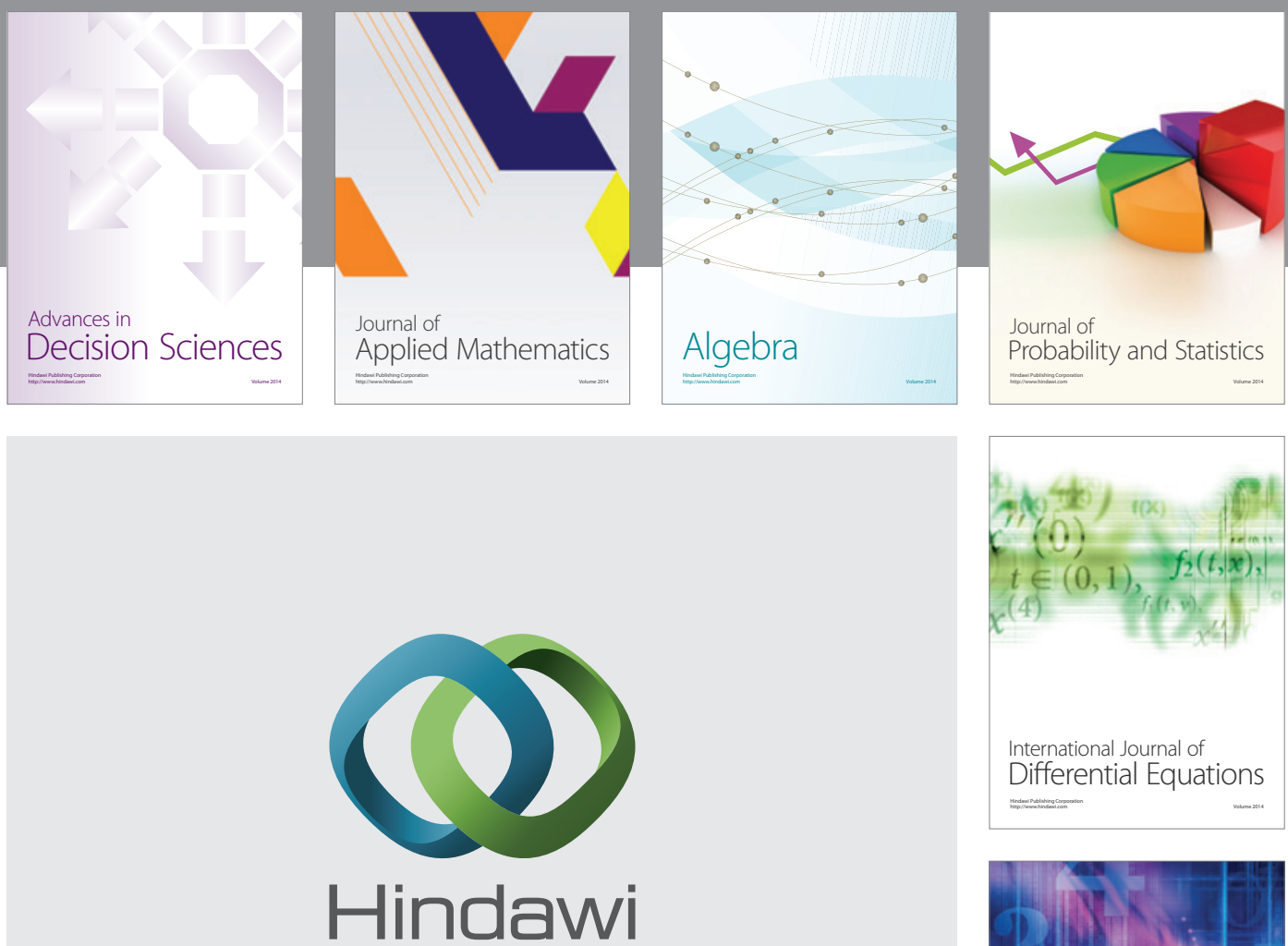

Submit your manuscripts at http://www.hindawi.com
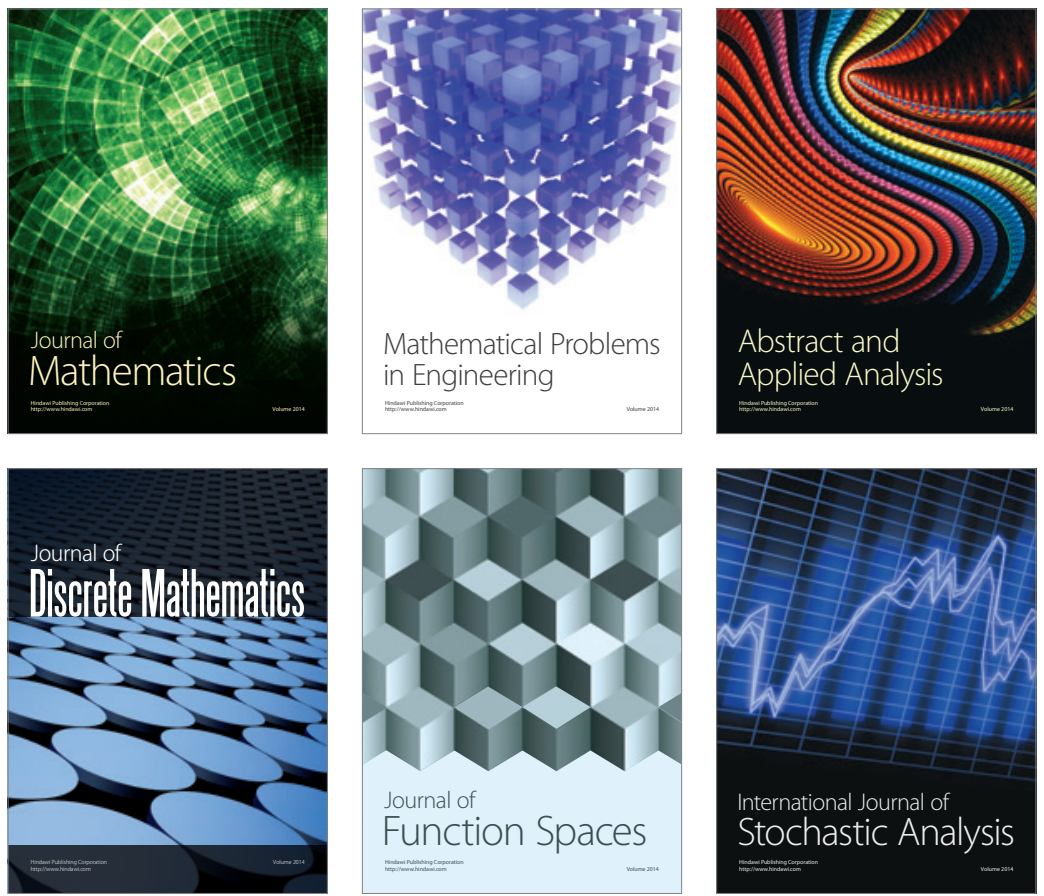

Journal of

Function Spaces

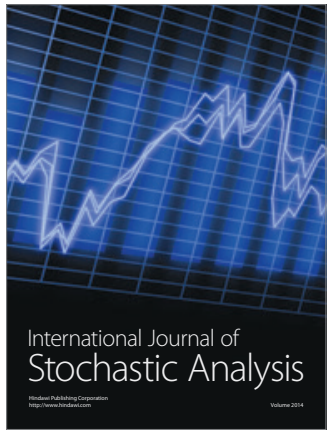

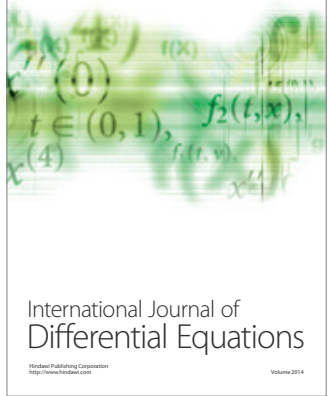
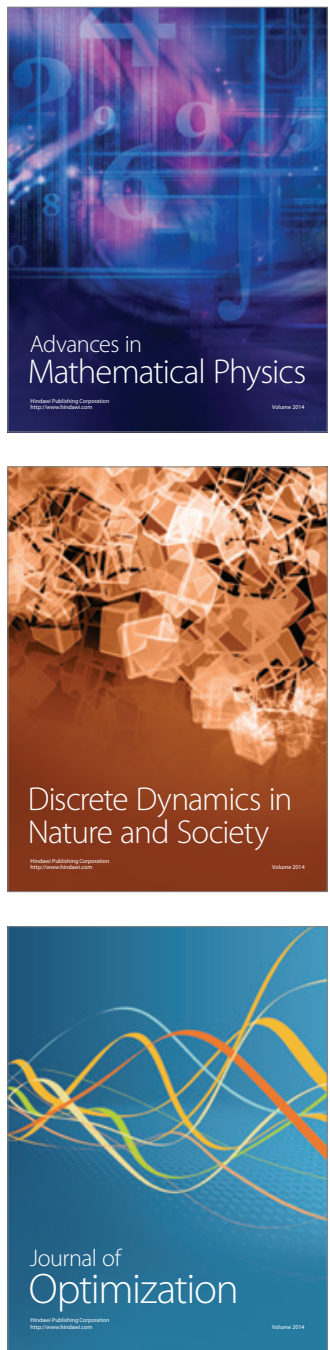